\title{
3-dimensional Bol loops as sections in non-solvable Lie groups
}

\author{
Ágota Figula
}

\begin{abstract}
Our aim in this paper is to classify the 3-dimensional connected differentiable global Bol loops, which have a non-solvable group as the group topologically generated by their left translations and to describe their relations to metric space geometries. The classification of global differentiable Bol loops significantly differs from the classification of local differentiable Bol loops. We treat the differentiable Bol loops as images of global differentiable sections $\sigma: G / H \rightarrow G$ such that for all $r, s \in \sigma(G / H)$ the element $r s r$ lies in $\sigma(G / H)$, where $H$ is the stabilizer of the identity $e$ of $L$ in $G$.
\end{abstract}

2000 Mathematics Subject Classification: 20N05, 22E99, 51H20, 53C35, 57N16.

Key words and phrases: differentiable Bol loops, affine symmetric spaces and Bruck loops, metric space geometry, differentiable sections in Lie groups.

\section{Introduction}

Differentiable diassociative loops can be treated following the ideas of Sophus Lie since for their tangential objects, binary Lie algebras, Lie's first theorem and Lie's third theorem are satisfied: To every binary Lie algebra there exists (up to local isomorphisms) a unique differentiable diassociative local loop. Kuzmin, Kerdman, and Nagy have proved that any local differentiable Moufang loop (these loops form a subclass of the class of diassociative loops) can uniquely be embedded into a connected simply connected global one (cf. [8], 12], [17]). This problem is more difficult for diassociative loops. Namely, there are local differentiable diassociative loops such that the corresponding global analytic loops do not exist (cf. 5]). In [5] the author gives in special cases the exact condition when a local diassociative loop can be embedded in a connected simply connected global one.

The present research on loops turns to such classes $\mathcal{C}$ of differentiable loops 
which have local forms determined in a unique way by algebras the properties of which are deduced by the properties of the tangential objects of loops in the class $\mathcal{C}$. The most important class $\mathcal{C}$ of such loops are the Bol loops. Their tangential objects, the Bol algebras, can be seen as Lie triple systems with an additional binary operation (cf. [18] pp. 84-86, Def. 6.10.). As known the Lie triple systems are in one-to-one correspondence to (global) simply connected symmetric spaces (cf. [14, [18 Sec. 6). Hence there is a strong connection between the theory of differentiable Bol loops and the theory of symmetric spaces. In particular the theory of connected differentiable Bruck loops ( which form a subclass of the class of Bol loops) is essentially the theory of affine symmetric spaces (cf. [18] Sec. 11).

The 1-dimensional connected differentiable Bol loops are isomorphic either to the group $\mathbb{R}$ or to $\mathrm{SO}_{2}(\mathbb{R})$. The smallest connected differentiable proper Bol loops are realized on 2-dimensional manifolds. There exist precisely two isotopism classes of proper 2-dimensional connected differentiable global Bol loops. As a representative of the one class we may choose the hyperbolic plane loop (cf. [18], Section 22); this loop is the unique Bruck loop in the isotopism class of loops, having the connected component of the group of hyperbolic motions as the group topologically generated by their left translations. As a representative of the other isotopism class may be chosen the 2-dimensional Bruck loop which is realized on the pseudo-euclidean affine plane such that its left translations group is the connected component of the group of pseudo-euclidean motions and the elements of $L$ are the lines of positive slope in the pseudo-euclidean affine plane (cf. [18] Section 25).

Our aim in this paper is to classify the 3-dimensional connected differentiable global Bol loops, which have a non-solvable group as the group topologically generated by their left translations and to describe their relations to metric space geometries. The classification of global differentiable Bol loops significantly differs from the classification of local differentiable Bol loops. There exist much more local differentiable Bol loops than global differentiable Bol loops, as we will also show in this paper.

We treat the differentiable Bol loops as images of global differentiable sections $\sigma: G / H \rightarrow G$ such that for all $r, s \in \sigma(G / H)$ the element $r s r$ lies in $\sigma(G / H)$, where $H$ is the stabilizer of the identity $e$ of $L$ in $G$. Using the present theory of differentiable Bol loops it is not difficult to prove that $G$ is four, five or sixdimensional. The images of sections for local Bol loops can be realized as the exponential images of Lie triple systems. But then we have to discuss which of these local Bol loops can be extended to global Bol loops. The results of our paper can summarized in the following

Theorem There are precisely two isotopism classes $\mathcal{C}_{i},(i=1,2)$ of the 
3-dimensional connected differentiable simple proper Bol loops $L$ such that the group $G$ generated by the left translations $\left\{\lambda_{x} ; x \in L\right\}$ is a non-solvable Lie group.

The class $\mathcal{C}_{1}$ consists of Bol loops having the simple Lie group $G=$ $P S L_{2}(\mathbb{C})$ as the group topologically generated by their left translations and as the stabilizer $H$ of $e \in L$ in $G$ the group $S_{3}(\mathbb{R})$.

Any loop in the class $\mathcal{C}_{1}$ can be characterized by a real parameter a, where $-1<a<1$. The loops $L_{a}$ are isotopic to the hyperbolic space loop $L_{0}$, which is realized on the hyperbolic space by the multiplication $x \cdot y=\tau_{e, x}(y)$, where $\tau_{e, x}$ is the hyperbolic translation moving e onto $x$. The loop $L_{0}$ is the only Bruck loop in $\mathcal{C}_{1}$. The loops $L_{a}$ and $L_{b}$ in $\mathcal{C}_{1}$ are isomorphic if and only if the angles between the tangent 3-space $\mathbf{m}_{\mathbf{a}}$, respectively $\mathbf{m}_{\mathbf{b}}$ and the tangent 3-space $\mathbf{m}_{0}$ are the same with respect to the Cartan-Killing form. This is the case if and only if $b= \pm a$.

The other class $\mathcal{C}_{2}$ of simple Bol loops consists of 3-dimensional connected differentiable Bol loops such that the group $G$ topologically generated by their left translations is the semidirect product $P S L_{2}(\mathbb{R}) \ltimes \mathbb{R}^{3}$, where the action of $P S L_{2}(\mathbb{R})$ on $\mathbb{R}^{3}$ is the adjoint action of $P S L_{2}(\mathbb{R})$ on its Lie algebra, and for the stabilizer $H$ of $e \in L$ we have

$$
\left\{\left( \pm\left(\begin{array}{rr}
\cos t & \sin t \\
-\sin t & \cos t
\end{array}\right),\left(\begin{array}{rr}
-x & y \\
y & x
\end{array}\right)\right) ; t \in[0,2 \pi], x, y \in \mathbb{R}\right\}
$$

Any loop in this class can be characterized by three real parameters $a, b, c$, with $a^{2}+b^{2}<1$. In $\mathcal{C}_{2}$ there is only one isomorphism class of Bruck loops, which consists of the loops $\hat{L}_{c}=L_{0,0, c}$. As a representative of this isomorphism class may be chosen the pseudo-euclidean space loop $\hat{L}=L_{0,0,0}$, which is realized on the pseudo-euclidean affine space. The connected component of the group of pseudo-euclidean motions is the group topologically generated by the left translations of $\hat{L}$. The elements of $\hat{L}$ are the planes on which the euclidean metric is induced. Two loops $L_{a, b, c}$ and $L_{a^{\prime}, b^{\prime}, c^{\prime}}$ in the class $\mathcal{C}_{2}$ are isomorphic if and only if the angles between the tangent 3-space $\mathbf{m}_{a, b, c}$, respectively $\mathbf{m}_{a^{\prime}, b^{\prime}, c^{\prime}}$ and the tangent 3 -space $\hat{\mathbf{m}}=\mathbf{m}_{0,0,0}$ are the same with respect to the Cartan-Killing form. This is the case if and only is $a= \pm a^{\prime}$. The non-simple 3-dimensional differentiable proper Bol loops are either the direct products of a 1-dimensional Lie group with a 2-dimensional Bol loop isotopic to the hyperbolic plane loop or the unique Scheerer extension of the Lie group $\mathrm{SO}_{2}(\mathbb{R})$ by the 2-dimensional hyperbolic plane loop.

Another impotant class of differentiable loops which generalize the class of abelian groups is the class of left A-loops. These loops correspond strongly to reductive homogeneous spaces. We show that any 3-dimensional differentiable Bol loop which is also a left A-loop is either a Bruck loop or the unique 
Scheerer extension of the orthogonal group $\mathrm{SO}_{2}(\mathbb{R})$ by the 2-dimensional hyperbolic plane loop (which is not a Bruck loop).

At the end of this paper we realize within the 3-dimensional pseudo-euclidean geometry on the set of euclidean planes differentiable loops which are not Bol loops but the corresponding section of which are given in a pure geometric manner.

\section{Bol loops and their Bol algebras}

A set $L$ with a binary operation $(x, y) \mapsto x \cdot y$ is called a loop if there exists an element $e \in L$ such that $x=e \cdot x=x \cdot e$ holds for all $x \in L$ and the equations $a \cdot y=b$ and $x \cdot a=b$ have precisely one solution which we denote by $y=a \backslash b$ and $x=b / a$. The left translation $\lambda_{a}: y \mapsto a \cdot y: L \rightarrow L$ is a bijection of $L$ for any $a \in L$. Two loops $\left(L_{1}, \circ\right)$ and $\left(L_{2}, *\right)$ are called isotopic if there are three bijections $\alpha, \beta, \gamma: L_{1} \rightarrow L_{2}$ such that $\alpha(x) * \beta(y)=\gamma(x \circ y)$ holds for any $x, y \in L_{1}$. An isotopism is an equivalence relation. If $\alpha=\beta=\gamma$ then the isotopic loops $\left(L_{1}, \circ\right)$ and $\left(L_{2}, *\right)$ are called isomorphic. Let $\left(L_{1}, \cdot\right)$ and $\left(L_{2}, *\right)$ be two loops. The direct product $L=L_{1} \times L_{2}=\left\{(a, b) \mid a \in L_{1}, b \in L_{2}\right\}$ with the multiplication $\left(a_{1}, b_{1}\right) \circ\left(a_{2}, b_{2}\right)=\left(a_{1} \cdot a_{2}, b_{1} * b_{2}\right)$ is again a loop, which is called the direct product of $L_{1}$ and $L_{2}$, and the loops $\left(L_{1}, \cdot\right),\left(L_{2}, *\right)$ are subloops of $(L, \circ)$.

A loop $L$ is called a Bol loop if for any two left translations $\lambda_{a}, \lambda_{b}$ the product $\lambda_{a} \lambda_{b} \lambda_{a}$ is again a left translation of $L$.

Let $G$ be the group generated by the left translations of $L$ and let $H$ be the stabilizer of $e \in L$ in the group $G$. The left translations of $L$ form a subset of $G$ acting on the cosets $\{x H ; x \in G\}$ such that for any given cosets $a H, b H$ there exists precisely one left translation $\lambda_{z}$ with $\lambda_{z} a H=b H$.

Conversely let $G$ be a group, $\mathrm{H}$ be a subgroup containing no normal nontrivial subgroup of $G$ and $\sigma: G / H \rightarrow G$ be a section such that $\sigma$ satisfies the following conditions:

1. The image $\sigma(G / H)$ forms a subset of $G$ with $\sigma(H)=1 \in G$.

2. $\sigma(G / H)$ generates $G$.

3. $\sigma(G / H)$ acts sharply transitively on the space $G / H$ of the left cosets $\{x H, x \in G\}$ (cf. [18], p. 18).

Then the multiplication on the factor space $G / H$ defined by $x H * y H=$ $\sigma(x H) y H$ yields a loop $L(\sigma)$. This loop is a Bol loop if and only if for all $r, s \in \sigma(G / H)$ the element $r s r$ is contained in $\sigma(G / H)$.

If $L_{1}$ and $L_{2}$ are Bol loops, then the direct product $L_{1} \times L_{2}$ is again a Bol loop.

Proposition 1. Let $L$ be a loop and let $G$ be the group generated by the 
left translations of $L$, and denote by $H$ the stabilizer of $e \in L$ in $G$. If $G$ and $H$ are direct products $G=G_{1} \times G_{2}$ and $H=H_{1} \times H_{2}$ with $H_{i} \subset G_{i}$ $(i=1,2)$ then the loop $L$ is the direct product of two loops $L_{1}$ and $L_{2}$, and $L_{i}$ is isomorphic to a loop $L_{i}^{*}$ having $G_{i}$ as the group generated by its left translations and $H_{i}$ as the corresponding stabilizer subgroup $(i=1,2)$.

In particular there is no 3-dimensional Bol loop $L$ such that $L$ is the direct product of a 1-dimensional and a 2-dimensional Bol loop and $G$ is a 5-or 6-dimensional Lie group.

Proof. The first assertion is the Proposition 1.18. in [18]. The second assertion follows from the fact that a 1-dimensional Bol loop is a Lie group. Hence the dimension of the group $G$ is at most 4 .

If the elements of $L$ are points of a differentiable manifold and the operations $(x, y) \mapsto x \cdot y, \quad(x, y) \mapsto x / y,(x, y) \mapsto x \backslash y: L \times L \rightarrow L$ are differentiable mappings then $L$ is called a differentiable loop. Moreover the manifold $L$ is parallelizable since the set of the left translations is sharply transitive. For a differentiable manifold $L$ the group of all autohomeomorphisms of $L$ becomes with respect to the compact-open topology a topological group. If $L$ is a Bol loop then the group $G$ topologically generated by the left translations within the group of autohomeomorphisms is a Lie group (cf. [18], p. 33; [16], pp. 414-416).

Every connected differentiable Bol loop is isomorphic to a Bol loop $L$ realized on the factor space $G / H$, where $G$ is a connected Lie group, $H$ is a connected closed subgroup containing no normal subgroup $\neq\{1\}$ of $G$ and the sharply transitive section $\sigma$ of $L$ is a differentiable map (cf. [18, p. 32).

Proposition 2. If $L$ a connected differentiable 3-dimensional Bol loop, then the pairs $(G, H)$ for the factor space $G / H$ must be different from the following cases:

a) $G=S L_{2}(\mathbb{C})$ or $P S L_{2}(\mathbb{C}), H \in\left\{U_{0}, U_{1}\right\}$ respectively $H \in\left\{U_{0} / \mathbb{Z}_{2}, U_{1} / \mathbb{Z}_{2}\right\}$ with $U_{r}=\left\{\left(\begin{array}{cc}z & (r-1) w \\ -(r+1) \bar{w} & \bar{z}\end{array}\right) ;|z|^{2}+\left(r^{2}-1\right)|w|^{2}=1\right\}$.

b) $G$ is locally isomorphic to $\mathrm{SO}_{3}(\mathbb{R}) \times \mathbb{R}$ and $H$ is a 1-dimensional subgroup of $G$.

c) The group $G=S O_{3}(\mathbb{R}) \ltimes \mathbb{R}^{3}$ is the connected component of the euclidean motion group and $H$ is a semidirect product of a 2-dimensional translation group $\mathbb{R}^{2}$ by a 1-dimensional rotation group $\mathrm{SO}_{2}(\mathbb{R})$.

d) $G=S_{3}(\mathbb{R}) \times S_{3}(\mathbb{R})$, and $H$ is any 3-dimensional subgroup of $G$.

e) $G=S L_{2}(\mathbb{C})$ or $P S L_{2}(\mathbb{C})$ and $H=W_{r}$ respectively $W_{r} \mathbb{Z}_{2} / \mathbb{Z}_{2}$, where $W_{r}=\left\{\left(\begin{array}{cc}\exp ((r i-1) x) & 0 \\ z & \exp (-(r i-1) x)\end{array}\right) ; x \in \mathbb{R}, z \in \mathbb{C}\right\}$ for $r \in \mathbb{R}$. 
Proof. In the case b) every 1-dimensional subgroup $H$ of $G$ containing no non-trivial normal subgroup of $G$ has one of the following shapes:

$$
H_{1}=\{K \times\{0\}\}, \quad \text { or } \quad H_{2}=\{K \times \varphi(K)\},
$$

where $K$ is isomorphic to $\mathrm{SO}_{2}(\mathbb{R})$ and $\varphi$ is a non-trivial homomorphism.

In the case d) every 3-dimensional subgroup $H$ of $G$, which does not contain any normal subgroup $\neq\{1\}$ of $G$ is conjugate to $\left\{(a, a) \mid a \in S O_{3}(\mathbb{R})\right\}$.

In the cases a), c) and for $H_{1}$ in the case b) the factor space $G / H$ is a topological product of spaces having as a factor the 2-sphere or the projective plane, which are non-parallelizable. In the cases d), e) and for $\mathrm{H}_{2}$ in the case b) the factor space $G / H$ is compact ([18], Section 16).

A real vector space $V$ with a trilinear multiplication $(., . .$.$) is called a Lie$ triple system $\mathcal{V}$, if the following identities are satisfied:

$$
\begin{gathered}
(\mathrm{X}, \mathrm{X}, \mathrm{Y})=0 \\
(\mathrm{X}, \mathrm{Y}, \mathrm{Z})+(\mathrm{Y}, \mathrm{Z}, \mathrm{X})+(\mathrm{Z}, \mathrm{X}, \mathrm{Y})=0 \\
(\mathrm{X}, \mathrm{Y},(\mathrm{U}, \mathrm{V}, \mathrm{W}))=((\mathrm{X}, \mathrm{Y}, \mathrm{U}), \mathrm{V}, \mathrm{W})+(\mathrm{U},(\mathrm{X}, \mathrm{Y}, \mathrm{V}), \mathrm{W})+(\mathrm{U}, \mathrm{V},(\mathrm{X}, \mathrm{Y}, \mathrm{W})) .
\end{gathered}
$$

A Bol algebra $A$ is a Lie triple system $(V,(., .,)$.$) with a bilinear skew-$ symmetric operation [[.,.]], $(X, Y) \mapsto[[X, Y]]: V \times V \rightarrow V$ such that the following identity is satisfied:

$$
\begin{gathered}
{[[(X, Y, Z), W]]-[[(X, Y, W), Z]]+(Z, W,[[X, Y]])} \\
-(X, Y,[[Z, W]])+[[[[X, Y]],[[Z, W]]]]=0 .
\end{gathered}
$$

With any connected differentiable Bol loop $L$ we can associate a Bol algebra in the following way: Let $G$ be the Lie group topologically generated by the left translations of $L$, and let $(\mathrm{g},[.,]$.$) be the Lie algebra of G$. Denote by $\mathbf{h}$ the Lie algebra of the stabilizer $H$ of $e \in L$ in $G$ and by $\mathbf{m}=T_{1} \sigma(G / H)$ the tangent space at $1 \in G$ of the image of the section $\sigma: G / H \rightarrow G$ corresponding to the Bol loop $L$. Then $\mathbf{g}=\mathbf{m} \oplus \mathbf{h},[[\mathbf{m}, \mathbf{m}], \mathbf{m}] \subseteq \mathbf{m}$ and $\mathbf{m}$ generates the Lie algebra $\mathbf{g}$. The subspace $\mathbf{m}$ is the tangent Bol algebra of the Bol loop $L$ the operations of which are defined by $(X, Y, Z) \mapsto[[X, Y], Z]$, $(X, Y) \mapsto[X, Y]_{\mathbf{m}}$, where $X, Y, Z$ are elements of the subspace $\mathbf{m}$ and $Z \mapsto Z_{\mathbf{m}}: \mathbf{g} \rightarrow \mathbf{m}$ is the projection of $\mathbf{g}$ onto $\mathbf{m}$ along the subalgebra $\mathbf{h}$. Miheev and Sabinin define for any Bol algebra $A$ the standard enveloping Lie algebra of $A$ (cf. [16], pp. 428-429). If $L$ is a connected differentiable Bol loop having a Bol algebra $A$ as its tangent Bol algebra, then the Lie algebra $\mathbf{g}$ of the Lie group $G$ topologically generated by the left translations of $L$ is isomorphic to the standard enveloping Lie algebra of $A$. If $A$ is a 
$n$-dimensional Bol algebra, then the dimension of its standard enveloping Lie algebra is at most $n+\frac{n(n-1)}{2}$ (cf. [16], pp. 428-430).

From every triple $((\mathbf{g},[.,]),. \mathbf{h}, \mathbf{m})$ we can construct in a canonical way a triple $\left(\left(\mathbf{g}^{*},[., .]^{*}\right), \mathbf{h}^{*}, \mathbf{m}\right)$ (cf. [16], pp. 424-425, and [18], Section 6). The Lie algebra $\left(\mathbf{g}^{*},[.,]^{*}\right)$ is the Lie algebra of the isometry group of a symmetric space and the Lie algebra $\mathbf{g}$ is an epimorphic image of $\mathbf{g}^{*}$. Moreover the subspace $\mathbf{m} \subset(\mathbf{g},[.,]$.$) determines a Lie triple system A:=(\mathbf{m},(., .,)$.$) ,$ with $(., .,)=.[[.,],$.$] . If the intersection of the centre of \mathbf{g}$ with $[\mathbf{m}, \mathbf{m}]$ is trivial then the Lie algebra $\mathbf{g}$ is an epimorphic image of the Lie algebra of the isometry group for the symmetric space belonging to the Lie triple system $A$. Hence if $\mathbf{g}=\mathbf{g}^{*}$ then $\mathbf{g}=\mathbf{m} \oplus[\mathbf{m}, \mathbf{m}]$.

Remark. Let $G$ be a connected Lie group and let $\mathbf{g}$ be its Lie algebra. For the classification of differentiable local Bol loops $L$ having $G$ as the group topologically generated by the left translations such that $\mathbf{g}=\mathbf{m} \oplus[\mathbf{m}, \mathbf{m}]$ for the subspace $\mathbf{m}$ corresponding to the tangential space of $L$ holds we may proceed in the following way: First we determine all symmetric space $\mathbf{m}$ having $G$ as the isometry group. After this we have to find for any symmetric space $\mathbf{m}$ all subalgebras $\mathbf{h}$ with $\mathbf{g}=\mathbf{m} \oplus \mathbf{h}$ such that $\mathbf{h}$ does not contain any non-trivial ideal of $\mathbf{g}$. For the classification up to isotopisms it is enough to take for $\mathbf{h}$ a suitable representative of the set $\left\{g^{-1} \mathbf{h} g ; g \in G\right\}$.

Since the Bol loops are strongly left alternative ( Definition 5.3. in [18]) every global Bol loop contains an exponential image of a complement $\mathbf{m}$ of the Lie algebra $\mathbf{h}$ of $H$ in the Lie algebra $\mathbf{g}$ of $G$, such that $\mathbf{m}$ generates $\mathbf{g}$ and satisfies the relation $[[\mathbf{m}, \mathbf{m}], \mathbf{m}] \subseteq \mathbf{m}$.

Lemma 3. Let $L$ be a differentiable loop and denote by $\mathbf{m}$ the tangent space of $L$ at $1 \in G$. Then $\mathbf{m}$ does not contain any element of $A d_{g} \mathbf{h}$ for some $g \in G$.

Proof. For $g \in G$ the group $H^{g}=g^{-1} H g$ is the stabilizer of the element $g^{-1}(e) \in L$ in $G$.

The conjugation $\sigma(G / H)^{g}=g^{-1} \sigma(G / H) g$ of the section $\sigma$ of $L$ with an element $g \in G$ defines again a sharply transitive section $x H \mapsto \sigma(x H)^{g}$ and the loop $L^{*}$ belonging to the triple $\left(G, H, \sigma(G / H)^{g}\right)$ is isotopic to $\mathrm{L}$ (cf. [18, Theorem 1.11 (iii) p. 22). Moreover this loop $L^{*}$ is isomorphic to $L=(G, H, \sigma)$ if and only if there is an automorphism of $G$ leaving $H$ invariant and mapping the section $\sigma$ corresponding to $L$ onto the section $\sigma(G / H)^{g}$ corresponding to $L^{*}$ (cf. [18], Theorem 1.11 (i) p. 21). 
A loop $L$ is called a left A-loop if each $\lambda_{x, y}=\lambda_{x y}^{-1} \lambda_{x} \lambda_{y}: L \rightarrow L$ is an automorphism of $L$. The group $G$ topologically generated by the left translations of $L$ is a Lie group (cf. [18, Proposition 5.20, p. 75). Let $\mathrm{g}$ be the Lie algebra of $G$ and let $\mathbf{h}$ be the Lie subalgebra of the stabilizer $H$ of $e \in L$ in $G$. Denote by $\sigma: x \mapsto \lambda_{x}: L \rightarrow G$ the section corresponding to $L$. Then the tangent space $\mathbf{m}=T_{1} \sigma(L)$ of the image of the section $\sigma$ at $1 \in G$ is complementary to $\mathbf{h}$ and satisfies the properties $\mathbf{m} \oplus \mathbf{h}=\mathbf{g}$ and $[\mathbf{h}, \mathbf{m}] \subseteq \mathbf{m}$ (cf. [18, Definition 5.18. and Proposition 5.20. pp. 74-75), i.e. $\mathrm{g}$ is reductive with respect to $(\mathbf{h}, \mathbf{m})$ in the sence of Kobayashi and Nomizu (cf. [11] Vol II, p. 190).

A differentiable loop $L$ is called a Bruck loop if there is an involutory automorphism $\sigma$ of the Lie algebra $\mathbf{g}$ of the connected Lie group $G$ generated by the left translations of $L$ such that the tangent space $T_{e}(L)=\mathbf{m}$ is the -1-eigenspace and the Lie algebra $\mathbf{h}$ of the stabilizer $H$ of $e \in L$ in $G$ is the +1 -eigenspace of $\sigma$.

\section{3-dimensional Lie triple systems}

Let $(\mathbf{m},(., .,)$.$) be a Lie triple system. Denote by \left(\mathbf{g}^{*},[.,].\right)$ the Lie algebra of the group of displacements of the symmetric space belonging to a Lie triple system. We classify the 3-dimensional non-solvable Lie triple systems $(\mathbf{m},(., .,)$.$) within the group \mathbf{g}^{*}$ as subspaces with $(., .,)=.[[.,],$.$] .$

a) The semisimple Lie triple systems

If $\mathbf{m}$ is a 3 -dimensional semisimple Lie triple system then the corresponding Lie algebra is semisimple with $4 \leq \operatorname{dim} \mathbf{g}^{*} \leq 6$ ([13, p. 219, and Theorem 2.7. p. 222). Every semisimple Lie algebra $\mathbf{g}^{*}$ has dimension 6 . To classify the 3 -dimensional semisimple Lie triple systems we have to determine all involutory automorphisms of the Lie algebra $\mathbf{g}^{*}$, which leave a 3-dimensional subalgebra of $\mathbf{g}^{*}$ elementwise fixed. This problem is equivalent to give all involutory automorphisms of the Lie group $G^{*}$, which fix elementwise a 3dimensional subgroup of $G^{*}$.

Any 6-dimensional semisimple Lie group is locally isomorphic to one of the following Lie groups:

1. $P S L_{2}(\mathbb{R}) \times S O_{3}(\mathbb{R})$

2. $P S L_{2}(\mathbb{R}) \times P S L_{2}(\mathbb{R})$

3. $\mathrm{SO}_{3}(\mathbb{R}) \times \mathrm{SO}_{3}(\mathbb{R})$

4. $P S L_{2}(\mathbb{C})$.

1. case: The automorphism group $\Gamma$ of the Lie group $G^{*}=P S L_{2}(\mathbb{R}) \times$ $S_{3}(\mathbb{R})$ is the direct product of the automorphism group of $P S L_{2}(\mathbb{R})$ and 
the automorphism group of $\mathrm{SO}_{3}(\mathbb{R})$. Since there is no involutory automorphism of $\Gamma$ leaving a 3-dimensional subgroup of $G^{*}$ elementwise fixed therefore there is no 3 -dimensional Lie triple system corresponding to the Lie group $G^{*}=P S L_{2}(\mathbb{R}) \times S O_{3}(\mathbb{R})$.

2. case: The automorphism group $\Gamma$ of the Lie group $G^{*}=P S L_{2}(\mathbb{R}) \times$ $P S L_{2}(\mathbb{R})$ is the semidirect product of the normal automorphism group $\Gamma_{1} \times \Gamma_{1}$, where $\Gamma_{1}$ is the automorphism group of $P S L_{2}(\mathbb{R})$, by the group generated by the automorphism $\sigma: G^{*} \rightarrow G^{*} ;(u, v) \mapsto(v, u)$. The only involutory automorphisms centralizing a 3-dimensional subgroup of $\Gamma$ are the conjugate elements to $\sigma$. Therefore there is up to conjugation precisely one 3-dimensional Lie triple system $\mathbf{m}$ having the shape $\left\{(X,-X) \mid X \in s l_{2}(\mathbb{R})\right\}$. 3. case: In the case $G^{*}=S O_{3}(\mathbb{R}) \times S O_{3}(\mathbb{R})$ there is up to conjugation only one Lie triple system corresponding to $G^{*}$. This Lie triple system has the form $\left\{(X,-X) \mid X \in \mathrm{so}_{3}(\mathbb{R})\right\}$.

4. case: B1 A real basis of the Lie algebra $s l_{2}(\mathbb{C})$ : Let us consider in $\mathrm{g}^{*}$ the following real basis $\{\mathcal{H}, \mathcal{T}, \mathcal{U}, \mathrm{i} \mathcal{H}, \mathrm{i} \mathcal{T}, \mathrm{i} \mathcal{U}\}$, where $\mathcal{H}=\left(\begin{array}{rr}1 & 0 \\ 0 & -1\end{array}\right)$, $\mathcal{T}=\left(\begin{array}{ll}0 & 1 \\ 1 & 0\end{array}\right), \mathcal{U}=\left(\begin{array}{rr}0 & 1 \\ -1 & 0\end{array}\right)$. The Lie algebra multiplication is given by the following rules: $[\mathcal{H}, \mathcal{T}]=2 \mathcal{U},[\mathcal{H}, \mathcal{U}]=2 \mathcal{T},[\mathcal{U}, \mathcal{T}]=2 \mathcal{H}$.

K1 The normalized complex Cartan-Killing form $k_{\mathbb{C}}: s l_{2}(\mathbb{C}) \times s l_{2}(\mathbb{C}) \rightarrow \mathbb{C}$ of $s_{2}(\mathbb{C})$ is the bilinear form defined by $k_{\mathbb{C}}(X, Y)=\frac{1}{8} \operatorname{trace}(\operatorname{ad} X \operatorname{ad} Y)$. If $X \in s_{2}(\mathbb{C})$ has the decomposition

$$
X=\lambda_{1} \mathcal{H}+\lambda_{2} \mathcal{T}+\lambda_{3} \mathcal{U}+\lambda_{4} \mathrm{i} \mathcal{H}+\lambda_{5} \mathrm{i} \mathcal{T}+\lambda_{6} \mathrm{i} \mathcal{U}
$$

then the complex Cartan-Killing form $k_{\mathbb{C}}$ satisfies

$$
k_{\mathbb{C}}(X)=\lambda_{1}^{2}+\lambda_{2}^{2}+\lambda_{6}^{2}-\lambda_{3}^{2}-\lambda_{4}^{2}-\lambda_{5}^{2}+i\left(2 \lambda_{1} \lambda_{4}+2 \lambda_{2} \lambda_{5}-2 \lambda_{3} \lambda_{6}\right)
$$

(cf. [20], Section 6.1, pp. 215-228). The normalized real Cartan-Killing form $k_{\mathbb{R}}: s l_{2}(\mathbb{C}) \times s l_{2}(\mathbb{C}) \rightarrow \mathbb{R}$ is the restriction of $k_{\mathbb{C}}$ to $\mathbb{R}$ such that $k_{\mathbb{R}}(X)=\lambda_{1}^{2}+\lambda_{2}^{2}+\lambda_{6}^{2}-\lambda_{3}^{2}-\lambda_{4}^{2}-\lambda_{5}^{2}$ and the basis $\{\mathcal{H}, \mathcal{T}, \mathcal{U}, \mathrm{i} \mathcal{H}, \mathrm{i} \mathcal{T}, \mathrm{i} \mathcal{U}\}$ is orthonormal with respect to $k_{\mathbb{R}}$.

The automorphism group $\Gamma$ of $P S L_{2}(\mathbb{C})$ is the semidirect product of the group of inner automorphisms by the group of order 2 generated by the outer automorphism $\tau: z \mapsto \bar{z}$. According to ([15], pp. 153-154) there are in $\Gamma$ precisely two conjugacy classes of involutory automorphisms centralizing 3 -dimensional subgroups. In the one class there is involutory automorphism fixing the group $\left\{\left(\begin{array}{rr}a & b \\ -\bar{b} & \bar{a}\end{array}\right) ; a, b \in \mathbb{C}, a \bar{a}+b \bar{b}=1\right\} / \mathbb{Z}_{2} \cong S O_{3}(\mathbb{R})$ elementwise. In the other conjugacy class there is an involutory automorphism centralizing the subgroup $\left\{\left(\begin{array}{ll}a & b \\ \bar{b} & \bar{a}\end{array}\right) ; a, b \in \mathbb{C}, a \bar{a}-b \bar{b}=1\right\} / \mathbb{Z}_{2} \cong P S L_{2}(\mathbb{R})$. 
4.1 The subalgebra $\mathbf{h}_{1}$ isomorphic to $s o_{3}(\mathbb{R})$ is generated by the basis elements $i \mathcal{H}, i \mathcal{T}, \mathcal{U}$. The Lie triple system $\mathbf{m}$ with the property $[\mathbf{m}, \mathbf{m}]=\mathbf{h}_{1}$ has as generators $\mathcal{H}, \mathcal{T}, \mathrm{i} \mathcal{U}$.

4.2 The subalgebra $\mathbf{h}_{2}$ isomorphic to $\operatorname{sl}_{2}(\mathbb{R})$ has as basis elements $\mathrm{i} \mathcal{H}, \mathcal{T}, \mathrm{i} \mathcal{U}$. The Lie triple system $\mathbf{m}$ with the property $[\mathbf{m}, \mathbf{m}]=\mathbf{h}_{2}$ is generated by the elements $\mathcal{H}, \mathrm{i} \mathcal{T}, \mathcal{U}$.

b) Since there is no non-solvable 3 -dimensional Lie triple system with a 2-dimensional radical $\mathbf{r}(\mathbf{m})$ (cf. [13, Theorem 2.20. p. 227) we have to consider Lie triple systems having a 1-dimensional radical.

Let the subspace $\mathbb{R} e_{1}$ be the 1-dimensional radical $\mathbf{r}(\mathbf{m})$ of the Lie triple system $\mathbf{m}$. One has $\mathbf{m}=\mathbf{r}(\mathbf{m}) \oplus \mathbf{u}_{\mathbf{0}}$, where $\mathbf{u}_{\mathbf{0}}$ is a 2-dimensional semisimple subsystem generated by $e_{2}$ and $e_{3}$. Since the isometry group of the symmetric space $\mathcal{P}$ corresponding to $u_{0}$ is 3 -dimensional we may assume that $\left[e_{2}, e_{3}\right]=e_{4}$ is the generator of the Lie algebra of the stabilizer of a point in $\mathcal{P}$.

According to Furness ( [4], pp. 44-45) we have 3 types of 2-dimensional semisimple Lie triple systems. The elements $e_{2}, e_{3}$ generate the Lie algebra of $\mathrm{SO}_{3}(\mathbb{R})$ if and only if

$$
\left[e_{4}, e_{2}\right]=e_{3}, \quad\left[e_{4}, e_{3}\right]=-e_{2} .
$$

The elements $e_{2}, e_{3}$ generate the Lie algebra of $P S L_{2}(\mathbb{R})$ if and only if either

$$
\left[e_{4}, e_{2}\right]=-e_{3}, \quad\left[e_{4}, e_{3}\right]=e_{2}
$$

and $e_{4}$ is elliptic with respect to the Cartan-Killing form, or

$$
\left[e_{4}, e_{2}\right]=-e_{3}, \quad\left[e_{4}, e_{3}\right]=-e_{2}
$$

and the element $e_{4}$ is hyperbolic.

Since $\mathbf{m}$ is a Lie triple system with the properties $\left[\left[\mathbf{m}, e_{1}\right], e_{1}\right]=0$ and $\left[\left[e_{1}, \mathbf{m}\right], \mathbf{m}\right] \subset\left\langle e_{1}\right\rangle$ we obtain that

1. $\left[\left[e_{1}, e_{2}\right], e_{2}\right]=a e_{1},\left[\left[e_{1}, e_{3}\right], e_{3}\right]=b e_{1},\left[\left[e_{1}, e_{2}\right], e_{3}\right]=c e_{1}$

$$
\left[\left[e_{1}, e_{3}\right], e_{2}\right]=d e_{1},\left[\left[e_{2}, e_{3}\right], e_{1}\right]=(d-c) e_{1},
$$

where $a, b, c, d$ are real parameters.

5. First let $\mathbb{R} e_{1}$ be the centre of $\mathbf{m}$. Then we have $\left[\left[e_{1}, \mathbf{m}\right], \mathbf{m}\right]=0$ and $a=b=c=d=0$. We obtain 3 types of Lie triple systems with 1-dimensional centre belonging to the different types of the 2-dimensional semisimple Lie triple systems. Now we characterize the corresponding Lie 
algebras $\mathbf{g}^{*}$.

5.1 The Lie algebra $\mathbf{g}^{*}$ is isomorphic to the Lie algebra $s o_{3}(\mathbb{R}) \oplus \mathbb{R}$. The multiplication table in $\mathbf{g}^{*}$ is defined by the following relations:

$$
\left[e_{4}, e_{2}\right]=e_{3}, \quad\left[e_{4}, e_{3}\right]=-e_{2}, \quad\left[e_{4}, e_{i}\right]=0 \quad(i=2,3,4) .
$$

There are precisely two Lie triple systems $\mathbf{m}_{1}, \mathbf{m}_{2}$, such that the corresponding Lie algebra $\mathbf{g}^{*}$ is isomorphic to the Lie algebra $s l_{2}(\mathbb{R}) \oplus \mathbb{R}$.

B2 A real basis of the Lie algebra $s l_{2}(\mathbb{R}) \oplus \mathbb{R}$ : Denote by $e_{1}$ the generator of the Lie algebra $\mathbb{R}$. Let $e_{2}=(\mathcal{H}, 0), e_{3}=(\mathcal{T}, 0), e_{4}=(\mathcal{U}, 0)$, with $\mathcal{H}, \mathcal{T}, \mathcal{U}$ defined in $\mathbf{B} 1$, be a real basis of $\operatorname{sl}_{2}(\mathbb{R}) \oplus\{0\}$. Then the multiplication in $\mathbf{g}^{*}$ is given by the following rules:

$$
\left[e_{2}, e_{3}\right]=e_{4},\left[e_{4}, e_{2}\right]=-e_{3},\left[e_{4}, e_{3}\right]=e_{2},\left[e_{1}, e_{i}\right]=0(i=2,3,4) .
$$

5.2 The Lie triple system $\mathbf{m}_{1}$ is generated by the basis elements $e_{1}, e_{2}, e_{4}$.

5.3 The Lie triple system $\mathbf{m}_{2}$ has as generators $e_{1}, e_{2}, e_{3}$.

If the centre of $\mathbf{m}$ is trivial then not all parameters $a, b, c, d$ in the system 1 ) of b) are zero. Using the third property in the definition of a Lie triple system and an automorphism $\varphi$ of the form: $\varphi\left(e_{1}\right)=\alpha e_{1}, \varphi\left(e_{2}\right)=\beta e_{2}+\gamma e_{3}$, $\varphi\left(e_{3}\right)=\delta e_{2}+\varepsilon e_{3}$, where $\beta \varepsilon-\gamma \delta \neq 0$, we have the following four cases:

6.1 The Lie triple system $\mathbf{m}_{1}$ is given as follows:

$$
\left[e_{4}, e_{2}\right]=e_{3},\left[e_{4}, e_{3}\right]=-e_{2},\left[\left[e_{1}, e_{2}\right], e_{2}\right]=-e_{1},\left[\left[e_{1}, e_{3}\right], e_{3}\right]=-e_{1} .
$$

The corresponding Lie algebra $\mathbf{g}^{*}$ is isomorphic to the Lie algebra $s o_{3}(\mathbb{R}) \ltimes \mathbb{R}^{3}$. Since $\mathbb{R} e_{2}, \mathbb{R} e_{3}, \mathbb{R} e_{4}$ correspond to 1 -dimensional rotations and $\left[\left[e_{1}, e_{3}\right], e_{2}\right]=$ $0,\left[\left[e_{1}, e_{2}\right], e_{3}\right]=0,\left[e_{4}, e_{1}\right]=0$ the vectors $\left[e_{1}, e_{3}\right],\left[e_{1}, e_{2}\right], e_{1}$ are the axes of the rotation groups corresponding to $e_{2}, e_{3}$ respectively $e_{4}$. Hence $e_{1}, e_{5}:=$ $\left[e_{1}, e_{3}\right], e_{6}:=\left[e_{1}, e_{2}\right]$ form a basis of the radical of $\mathbf{g}^{*} \cong \mathbb{R}^{3}$. We have $\left[e_{4}, e_{6}\right]=$ $e_{5},\left[e_{4}, e_{5}\right]=-e_{6}$ because the rotation group belonging to $e_{4}$ with the axis $e_{1}$ leaves the 2-dimensional subspace $\left\langle e_{5}, e_{6}\right\rangle$ orthogonal to $\left\langle e_{1}\right\rangle$ invariant. Similarly the rotation groups corresponding to $e_{2}$ and to $e_{3}$ with axes $e_{5}$ respectively $e_{6}$ leaves the subspaces $\left\langle e_{1}, e_{6}\right\rangle$, respectively $\left\langle e_{1}, e_{5}\right\rangle$ invariant. Hence we have $\left[e_{2}, e_{6}\right]=e_{1},\left[e_{3}, e_{5}\right]=e_{1}$. Therefore the multiplication in the Lie algebra $\mathbf{g}^{*}$ is determined.

B3 Representing the Lie algebra $\mathbf{g}^{*}$ in the Lie algebra of real $(4 \times 4)$-matrices we may choose as basis the following matrices:

$$
e_{1}=\left(\begin{array}{rrrr}
0 & 0 & -1 & 0 \\
0 & 0 & 0 & 0 \\
0 & 0 & 0 & 0 \\
0 & 0 & 0 & 0
\end{array}\right), e_{2}=\left(\begin{array}{rrrr}
0 & 0 & 0 & 0 \\
0 & 0 & 0 & 0 \\
0 & 0 & 0 & -1 \\
0 & 0 & 1 & 0
\end{array}\right), e_{3}=\left(\begin{array}{rrrr}
0 & 0 & 0 & 0 \\
0 & 0 & 1 & 0 \\
0 & -1 & 0 & 0 \\
0 & 0 & 0 & 0
\end{array}\right),
$$




$$
e_{4}=\left(\begin{array}{rrrr}
0 & 0 & 0 & 0 \\
0 & 0 & 0 & 1 \\
0 & 0 & 0 & 0 \\
0 & -1 & 0 & 0
\end{array}\right), e_{5}=\left(\begin{array}{llll}
0 & 1 & 0 & 0 \\
0 & 0 & 0 & 0 \\
0 & 0 & 0 & 0 \\
0 & 0 & 0 & 0
\end{array}\right), e_{6}=\left(\begin{array}{llll}
0 & 0 & 0 & 1 \\
0 & 0 & 0 & 0 \\
0 & 0 & 0 & 0 \\
0 & 0 & 0 & 0
\end{array}\right)
$$

6.2

$$
\left[e_{4}, e_{2}\right]=-e_{3},\left[e_{4}, e_{3}\right]=e_{2},\left[\left[e_{1}, e_{2}\right], e_{2}\right]=e_{1},\left[\left[e_{1}, e_{3}\right], e_{3}\right]=e_{1} .
$$

6.3 The Lie triple $\mathbf{m}_{3}$ is given by the following relations:

$$
\left[e_{4}, e_{2}\right]=-e_{3},\left[e_{4}, e_{3}\right]=-e_{2},\left[\left[e_{1}, e_{2}\right], e_{2}\right]=e_{1},\left[\left[e_{1}, e_{3}\right], e_{3}\right]=-e_{1} .
$$

In both cases the Lie algebra $\mathbf{g}^{*}$ is isomorphic to $s l_{2}(\mathbb{R}) \ltimes \mathbb{R}^{3}$. From the relation in $\mathbf{6 . 3}$ we can deduce the multiplication table of the Lie algebra $\mathbf{g}^{*}$ :

$$
\begin{gathered}
{\left[e_{1}, e_{2}\right]=: e_{6},\left[e_{1}, e_{3}\right]=: e_{5},\left[e_{2}, e_{3}\right]=: e_{4},\left[e_{5}, e_{4}\right]=-e_{6},} \\
{\left[e_{1}, e_{4}\right]=\left[e_{1}, e_{5}\right]=\left[e_{1}, e_{6}\right]=\left[e_{2}, e_{5}\right]=\left[e_{3}, e_{6}\right]=\left[e_{6}, e_{5}\right]=0,} \\
{\left[e_{2}, e_{6}\right]=\left[e_{3}, e_{5}\right]=-e_{1},\left[e_{2}, e_{4}\right]=e_{3},\left[e_{3}, e_{4}\right]=-e_{2},\left[e_{6}, e_{4}\right]=e_{5} .}
\end{gathered}
$$

B4 Representing $\mathbf{g}^{*}$ as pairs of real $(2 \times 2)$-matrices we may choose as basis $e_{1}=(0,-\mathcal{U}), e_{2}=(\mathcal{H}, 0), e_{3}=(\mathcal{T}, 0), e_{4}=(\mathcal{U}, 0), e_{5}=(0,-\mathcal{H}), e_{6}=(0, \mathcal{T})$ with $\mathcal{H}, \mathcal{T}, \mathcal{U}$ defined in $\mathbf{B} 1$. The Lie triple system $\mathbf{m}_{2}$ defined by the relations 6.2 in the basis $\mathbf{B} 4$ is generated by the basis elements $e_{2}, e_{4}, e_{6}$. The Lie triple system $\mathbf{m}_{3}$ defined by the relations $\mathbf{6 . 3}$ belonging to the basis $\mathbf{B} 4$ has as generators $e_{1}, e_{2}, e_{3}$.

If an element $X$ of $\mathbf{g}^{*}=s l_{2}(\mathbb{R}) \ltimes \mathbb{R}^{3}$ has the decomposition

$$
X=\lambda_{1} e_{1}+\lambda_{2} e_{2}+\lambda_{3} e_{3}+\lambda_{4} e_{4}+\lambda_{5} e_{5}+\lambda_{6} e_{6}
$$

then the Cartan-Killing form $k$ (cf. [20], p.193) on $\mathbf{g}^{*}$ satisfies the relation

$$
\text { K2: } \quad k(X, X)=\lambda_{2}^{2}+\lambda_{3}^{2}-\lambda_{4}^{2} .
$$

7 The last Lie triple system and the corresponding Lie algebra $\mathbf{g}^{*}$ are defined by the following rules:

$$
\begin{gathered}
{\left[e_{4}, e_{2}\right]=-e_{3},\left[e_{4}, e_{3}\right]=-e_{2},\left[\left[e_{1}, e_{2}\right], e_{2}\right]=\frac{1}{4} e_{1},\left[\left[e_{1}, e_{3}\right], e_{3}\right]=-\frac{1}{4} e_{1},} \\
{\left[\left[e_{1}, e_{2}\right], e_{3}\right]=\frac{1}{4} e_{1},\left[\left[e_{1}, e_{3}\right], e_{2}\right]=-\frac{1}{4} e_{1},\left[\left[e_{2}, e_{3}\right], e_{1}\right]=-\frac{1}{2} e_{1} .}
\end{gathered}
$$

In this case the Lie algebra $\mathbf{g}^{*}$ is isomorphic to $s l_{2}(\mathbb{R}) \ltimes \mathbb{R}^{2}$. Moreover the elements $e_{2}, e_{3}$ and $e_{4}=\left[e_{2}, e_{3}\right]$ form a basis of $s l_{2}(\mathbb{R})$, such that $e_{2}$ is a hyperbolic, $e_{3}$ an elliptic and $e_{4}$ again a hyperbolic element with repect to the Cartan-Killing form. 


\section{Bol loops having semisimple Lie groups as the groups generated by their left transla- tions}

In this section we classify all 3-dimensional connected differentiable Bol loops having semisimple Lie groups as the groups topologically generated by their left translations and describe the symmetric spaces as well as the natural geometries associated with these loops.

Let $L$ be a 3 -dimensional proper Bol loop, let $G$ be the group generated by the left translations of $L$ and $H$ be the stabilizer of $e \in L$ in $G$. Since $G$ is semisimple and $4 \leq \operatorname{dim} G \leq 6$ we have $\operatorname{dim} G=6$.

Proposition 4. Let $G=G_{1} \times G_{2}$ be topologically generated by the left translations of a 3-dimensional connected differentiable Bol loop $L$ such that $G_{i}$ $(i=1,2)$ are 3 -dimensional quasi-simple Lie groups. Then $G_{i}(i=1,2)$ is isomorphic to $P S L_{2}(\mathbb{R})$ and the stabilizer $H$ of $e \in L$ in $G$ may be chosen either as

or

$$
\begin{gathered}
H_{1}=\left\{(x, x) \mid x \in P S L_{2}(\mathbb{R})\right\} \\
H_{2}=\left\{\left(\left(\begin{array}{ll}
a & b_{1} \\
0 & a^{-1}
\end{array}\right),\left(\begin{array}{ll}
a & b_{2} \\
0 & a^{-1}
\end{array}\right)\right) ; a>0, b_{1}, b_{2} \in \mathbb{R}\right\} .
\end{gathered}
$$

Proof. Denote by $\pi_{i}: G \rightarrow G_{i}$ the natural projection of $G$ to $G_{i}$ for $i=1,2$. Let $\operatorname{dim} \pi_{1}(H) \leq 1$. Since $H \leq \pi_{1}(H) \times \pi_{2}(H)$ one has $\operatorname{dim} \pi_{2}(H) \geq 2$. If $\operatorname{dim} \pi_{2}(H)=2$ then $H$ is the direct product of $\pi_{1}(H)$ with $\pi_{2}(H)$ and we obtain a contradiction to Proposition 1. Let $\pi_{2}(H)=G_{2}$. If $\operatorname{dim} \pi_{1}(H)=0$ then $H=\{1\} \times G_{2}$ which is impossible. If $\pi_{1}(H)$ is a 1-dimensional subgroup then $H$ would be 4-dimensional since there is no non-trivial homomorphism from $G_{2}$ into $\pi_{1}(H)$.

Let now $\operatorname{dim} \pi_{1}(H)=2$. We may assume that $\operatorname{dim} \pi_{2}(H) \geq 2$ since interchanging the indices we would obtain the previous case. Therefore each of the factors of $G$ is locally isomorphic to the group $P S L_{2}(\mathbb{R})$. Since the Lie algebra of $G_{2}$ is simple there is no non-trivial homomorphism from $\pi_{2}(H)=G_{2}$ into $\pi_{1}(H)$, so $\pi_{2}(H)$ cannot be $G_{2}$. If $\operatorname{dim} \pi_{2}(H)=\operatorname{dim} \pi_{1}(H)=2$ then $\pi_{i}(H) \cong \mathcal{L}_{2}=\{a x+b \mid a>0, b \in \mathbb{R}\}(i=1,2)$ and there exist homomorphisms $\varphi_{1}, \varphi_{2}$ with 1-dimensional kernels, such that $\varphi_{1}: \pi_{1}(H) \rightarrow \pi_{2}(H)$ and $\varphi_{2}: \pi_{2}(H) \rightarrow \pi_{1}(H)$. Since $H \cap G_{i}=\operatorname{ker} \varphi_{i}=\left\{\left(\begin{array}{ll}1 & b \\ 0 & 1\end{array}\right), b \in \mathbb{R}\right\}$ and (up to conjugation) $\operatorname{im} \varphi_{i}=\left\{\left(\begin{array}{ll}a & 0 \\ 0 & a^{-1}\end{array}\right), a>0\right\}(i=1,2)$ we obtain that

$$
\pi_{1}(H)=\operatorname{ker} \varphi_{1} \operatorname{im} \varphi_{2}, \pi_{2}(H)=\operatorname{ker} \varphi_{2} \operatorname{im} \varphi_{1}
$$


and $H$ has the shape (ii).

Finally let $\operatorname{dim} \pi_{1}(H)=3$. From the previous arguments it follows that $\operatorname{dim} \pi_{2}(H)=3$. If there is no homomorphism $\varphi: G_{1} \rightarrow G_{2}$ then there is no 3-dimensional subgroup $H$ of $G=G_{1} \times G_{2}$. If there exists a homomorphism $\varphi: G_{1} \rightarrow G_{2}$ then the stabilizer $H$ has the shape $\left\{(x, \varphi(x)) \mid x \in G_{1}\right\}$. Moreover $\varphi$ must be an isomorphism since otherwise $G$ would contain a discrete central subgroup of $G$. Hence $G$ can be identified with $G_{1} \times G_{1}$, where $G_{1}$ is isomorphic either to $P S L_{2}(\mathbb{R})$ or $S O_{3}(\mathbb{R})$, and $H$ may be chosen as the diagonal subgroup $\left\{(x, x) \mid x \in G_{1}\right\}$. According to Proposition $\left.2 \mathrm{~d}\right)$ we have $G=P S L_{2}(\mathbb{R}) \times P S L_{2}(\mathbb{R})$.

Now let $G$ be locally isomorphic to the group $P S L_{2}(\mathbb{C})$. According to ( [1], pp. 273-278) there are 4 conjugacy classes of the 3-dimensional subgroups of $G=S L_{2}(\mathbb{C})$, which we denote by $W_{r}, U_{0}, U_{1}$ and $S U_{2}(\mathbb{C})$. Since $S U_{2}(\mathbb{C})$ contains central elements $\neq 1$ of $S L_{2}(\mathbb{C})$ it follows from Proposition 2 that no of these groups can be the stabilizer of $e \in L$ in $G$. Hence it is sufficient to consider the following cases:

1. $G \cong P S L_{2}(\mathbb{R}) \times P S L_{2}(\mathbb{R})$, and $H_{i}(i=1,2)$ is one of the subgroups given in Proposition 4.

2. $G$ is isomorphic to $P S L_{2}(\mathbb{C})$ and $H$ is isomorphic to $S O_{3}(\mathbb{R})$.

Now we deal with the case 1 ). There is up to isomorphism precisely one Lie triple system $\mathbf{m}=\left\{(X,-X) \mid X \in \mathrm{sl}_{2}(\mathbb{R})\right\}$ corresponding to the Lie group $P S L_{2}(\mathbb{R}) \times P S L_{2}(\mathbb{R})($ 2. case, Section 3$)$.

The Lie algebra of $H_{1}$ is $\mathbf{h}_{1}=\left\{(X, X) \mid X \in s l_{2}(\mathbb{R})\right\}$. The Lie algebra $\mathbf{h}_{2}$ of $H_{2}$ is generated by the basis elements: $(\mathcal{H}, \mathcal{H}),(\mathcal{U}+\mathcal{T}, 0),(0, \mathcal{U}+\mathcal{T})$, where $\mathcal{H}, \mathcal{U}, \mathcal{T}$ are $(2 \times 2)$-matrices defined in B1. We see that the intersection of $\mathbf{m}$ and $\mathbf{h}_{1}$ is trivial. But the subalgebra $\mathbf{h}_{1}$ contains the element $(\mathcal{H}, \mathcal{H})$, which is conjugate to the element $(\mathcal{H},-\mathcal{H}) \in \mathbf{m}$ under the element corresponding to the pair of matrices $\left( \pm 1, \pm\left(\begin{array}{rr}0 & 1 \\ -1 & 0\end{array}\right)\right)$. This contradicts Lemma 3 .

Since the intersection of $\mathbf{m}$ and $\mathbf{h}_{2}$ is $\langle(\mathcal{U}+\mathcal{T},-(\mathcal{U}+\mathcal{T}))\rangle$ the subspace $\mathbf{m}$ cannot be a complement to $\mathbf{h}_{2}$ in $\mathbf{g}$.

From this discussion it follows that there is no 3-dimensional connected differentiable Bol loop such that the group $G$ generated by its left translations is isomorphic to the group $P S L_{2}(\mathbb{R}) \times P S L_{2}(\mathbb{R})$.

Now we consider the case 2. The Lie algebra $\mathbf{h}$ of $H$ with respect to the basis B1 (4.case, Section 3) has as generators $\mathcal{U}, \mathrm{i} \mathcal{T}, \mathrm{i} \mathcal{H}$. The Lie triple system $\mathbf{m}=\langle\mathrm{i} \mathcal{T}, \mathcal{U}, \mathcal{H}\rangle$ given in $\mathbf{4} .2$ cannot be a complement to $\mathbf{h}$ in $\mathbf{g}$ since $\mathbf{h} \cap \mathbf{m}=\langle\mathrm{i} \mathcal{T}, \mathcal{U}\rangle$. 
The Lie triple system $\mathbf{m}$ of $\mathbf{4 . 1}$ satisfies $[\mathbf{m}, \mathbf{m}]=\mathbf{h}$, and $\mathbf{g}=\mathbf{m} \oplus[\mathbf{m}, \mathbf{m}]$. Hence it determines a 3-dimensional Riemann symmetric space (cf. [11], Chapter VI, Theorem 2.2 (iii)). According to Theorem 11.8 in ([18]) there exists a global differentiable Bruck loop $L_{0}$ homeomorphic to $\mathbb{R}^{3}$ and having $G \cong P S L_{2}(\mathbb{C})$ as the group topologically generated by its left translations such that $H \cong S O_{3}(\mathbb{R})$ is the stabilizer of $e \in L$ in $G$. Since the symmetric spaces with $P S L_{2}(\mathbb{C})$ as the group of displacements which correspond to global differentiable Bol loops are isomorphic, there is precisely one isotopism class $\mathcal{C}$ of differentiable Bol loops having $P S L_{2}(\mathbb{R})$ as the group topologically generated by their left translations (cf. Remark in section 3 ) and $L_{0}$ is a representative of $\mathcal{C}$. Since the subspace $\mathbf{m}$ corresponding to $L_{0}$ is the hyperbolic space we call $L_{0}$ the hyperbolic space loop.

In order to determine within the isotopism class $\mathcal{C}$ the isomorphism classes we look for a suitable parametrization of the local Bol loops isotopic to the hyperbolic space loop. For this reason we consider all 3-dimensional complements to $\mathbf{h}=s_{3}(\mathbb{R})$ in $\mathbf{g}=s_{2}(\mathbb{C})$ with the properties $\mathbf{g}=\mathbf{m} \oplus \mathbf{h}$, $[[\mathbf{m}, \mathbf{m}], \mathbf{m}] \subseteq \mathbf{m}$ and $\mathbf{m}$ generates $\mathbf{g}$. We can write $\mathbf{m}$ in the general form:

$\mathbf{m}=\langle\mathcal{T}+\mathrm{a} \mathcal{U}+\mathrm{b} i \mathcal{T}+\mathrm{c} i \mathcal{H}, \mathrm{i} \mathcal{U}+\mathrm{d} \mathcal{U}+\mathrm{e} i \mathcal{T}+\mathrm{f} i \mathcal{H}, \mathcal{H}+\mathrm{g} \mathcal{U}+\mathrm{h} i \mathcal{T}+\mathrm{ki} \mathcal{H}\rangle$, $a, b, c, d, e, f, g, h, k \in \mathbb{R}$. According to [21] (pp. 217-219) there are precisely two classes of the Bol complements $\mathbf{m}$ to $\mathbf{h}$ in $\mathbf{g}$ having the following shapes: $\mathbf{m}_{a}=\langle\mathcal{T}+\mathrm{a} \mathcal{U}, \mathrm{i} \mathcal{U}+\mathrm{a}$ i $\mathcal{T}, \mathcal{H}\rangle$ for $a \in \mathbb{R} \backslash\{1,-1\}$,

$\mathbf{m}_{d}=\langle\mathcal{U}+\mathcal{T}, \mathrm{d} \mathrm{i} \mathcal{H}+\mathrm{i}(\mathcal{U}+\mathcal{T}), \mathcal{H}+\mathrm{d} \mathcal{U}\rangle$ for $d \in \mathbb{R} \backslash\{0\}$.

A Bol complement belongs to a local Bol loop in the class $\mathcal{C}$ if and only if $[\mathbf{m}, \mathbf{m}]$ is a compact subalgebra of $\mathbf{g}$. Since the value of the complex CartanKilling form $\mathbf{K} \mathbf{1}$ on the element $[\mathcal{U}+\mathcal{T}, \mathrm{d} \mathbf{i} \mathcal{H}+\mathrm{i}(\mathcal{U}+\mathcal{T})] \in\left[\mathbf{m}_{d}, \mathbf{m}_{d}\right]$ is zero $\left[\mathbf{m}_{d}, \mathbf{m}_{d}\right]$ is not compact. The subalgebra $\left[\mathbf{m}_{a}, \mathbf{m}_{a}\right]$ is compact if and only if $a \in(-1,1)$ since $\left[\mathbf{m}_{a}, \mathbf{m}_{a}\right]=\langle\mathrm{i} \mathcal{H}, \mathcal{U}+\mathrm{a} \mathcal{T}, \mathrm{i} \mathcal{T}+\mathrm{a} \mathrm{i} \mathcal{U}\rangle$.

In the isotopism class $\mathcal{C}=\left\{L_{a}, a \in(-1,1)\right\}$ only the Bol complement $\mathbf{m}_{0}$ satisfies the relation $\left[\mathbf{h}, \mathbf{m}_{0}\right] \subseteq \mathbf{m}_{0}$. Therefore only the hyperbolic space loop $L_{0}$ corresponding to the triple $\left(G, H\right.$, $\left.\exp \mathbf{m}_{0}\right)$ is a left A-loop. It is the unique Bruck loop in the class $\mathcal{C}$.

The complement $\mathbf{m}_{0}=\langle\mathcal{T}, \mathbf{i} \mathcal{U}, \mathcal{H}\rangle$ is orthogonal to $\mathbf{h}$ with respect to the Cartan-Killing form $k_{\mathbb{R}}$ on $\mathbf{g}$ (see $\mathbf{K} \mathbf{1}$ in Section 3). Let $\mathbf{m}_{a} \subset \mathbf{g}=\operatorname{sl}_{2}(\mathbb{C}$ ) be the tangent space $T_{1} \sigma_{a}(G / H)$ of the image $\sigma_{a}(G / H)$ of the differentiable section $\sigma_{a}: G / H \rightarrow G$ belonging to the loop $L_{a}$ for $a \in(-1,1)$. Two loops corresponding to $\left(G, H, \exp \mathbf{m}_{a}\right)$ and $\left(G, H, \exp \mathbf{m}_{b}\right)$ are isomorphic if and only if there exists an automorphism $\alpha$ of $\mathbf{g}$ such that $\alpha\left(\mathbf{m}_{a}\right)=\mathbf{m}_{b}$ and $\alpha(\mathbf{h})=\mathbf{h}$. The automorphism group of $\mathbf{g}$ leaving $\mathbf{m}_{0}$ and $\mathbf{h}$ invariant is the semidirect product $\Theta$ of $\mathrm{Ad}_{H}$ by the group generated by the involutory map $\varphi: z \mapsto \bar{z}$. The Cartan-Killing form $k_{\mathbb{R}}$ is invariant under $\Theta$. Hence the 3 
angles between $\mathbf{m}_{\mathbf{a}}$ and $\mathbf{m}_{\mathbf{0}}$ respectively between $\mathbf{m}_{\mathbf{b}}$ and $\mathbf{m}_{\mathbf{0}}$ are equal with respect to $k_{\mathbb{R}}$. The loops $L_{a}$ and $L_{b}$ are isomorphic if there exists a matrix $B=\left(\begin{array}{rr}c_{1}+c_{2} i & d_{1}+d_{2} i \\ -d_{1}+d_{2} i & c_{1}-c_{2} i\end{array}\right) \in H$ such that $\varphi\left(B^{-1} \mathbf{m}_{\mathbf{a}} B\right)=\mathbf{m}_{\mathbf{b}}$. This condition is equivalent with the following equations:

$$
\begin{aligned}
c_{1}^{2}+c_{2}^{2}+d_{1}^{2}+d_{2}^{2}=1, \quad d_{1} c_{2}-c_{1} d_{2}=0, \quad d_{1} c_{1}+c_{2} d_{2}=0, \\
c_{1} d_{2}+c_{2} d_{1}=0, \quad c_{2} d_{2}-c_{1} d_{1}=0, \\
\left(d_{2}^{2}-d_{1}^{2}-a\left(c_{2}^{2}-c_{1}^{2}\right)\right) b=c_{1}^{2}-c_{2}^{2}-a\left(d_{2}^{2}-d_{1}^{2}\right), \\
\left(d_{1} d_{2}+c_{1} c_{2} a\right) b=c_{1} c_{2}-a d_{1} d_{2}, \\
b\left(-a\left(c_{1}^{2}-c_{2}^{2}\right)+\left(d_{2}^{2}-d_{1}^{2}\right)\right)=-a\left(d_{2}^{2}-d_{1}^{2}\right)-\left(c_{1}^{2}-c_{2}^{2}\right), \\
b\left(c_{1} c_{2} a-d_{1} d_{2}\right)=d_{1} d_{2} a+c_{1} c_{2} .
\end{aligned}
$$

The solution of this system of equations is $b=\frac{1}{a}$ and $b=-a$. Since $b, a \in$ $(-1,1)$ it follows that $b=-a$. Therefore the loops $L_{a}, a \in[0,1)$ can be chosen as the representatives of the isomorphism classes of the Bol loops $L_{a}, a \in(-1,1)$.

The multiplication of the hyperbolic space loop $L_{0}$ may be given in the 3 dimensional hyperbolic geometry $\mathbb{H}_{3}$ explicitly. We choose a point $e$ in $\mathbb{H}_{3}$ and define $x \circ y=\tau_{e, x}(y)$ for all points $x, y \in \mathbb{H}_{3}$, where $\tau_{e, x}$ is the hyperbolic translation along the line joining $e$ with $x$ and mapping $e$ onto $x$.

The hyperbolic space geometry has an elementary model in the upper half space $\mathbb{R}^{3+}=\left\{(x, y, z) \in \mathbb{R}^{3} ; z>0\right\}$. We can identificate the elements of $\mathbb{R}^{3}$ with the elements of the $\mathbf{J}$-quaternion space. The $\mathbf{J}$-quaternion space is the 3 -dimensional subspace of the quaternion space which is orthogonal to the canonical basis quaternion $k$ (cf. [3], p. 3). We can give the action of the group $S L_{2}(\mathbb{C})$ on $\mathbb{R}^{3}$ by the linear rational functions: $\gamma(w)=(a w+b)(c w+$ $d)^{-1}$, where $\gamma=\left(\begin{array}{ll}a & b \\ c & d\end{array}\right), a, b, c, d \in \mathbb{C}, a d-b c=1, w=x+j y \in \mathbf{J}, x \in \mathbb{C}$, $y \in \mathbb{R}$ (cf. [3], p. 26). The restriction of this action onto the subspace $\mathbb{R}^{3+}$ defines the action of $S L_{2}(\mathbb{C})$ on the upper half space, and $(w, \pm \gamma) \mapsto$ $\gamma(w)$ is the transitive action of $P S L_{2}(\mathbb{C})$ on the upper half space. Since the stabilizer subgroup $\mathrm{H}=\mathrm{SO}_{3}(\mathbb{R})$ leaves the point $j$ fixed, we may choose this point as the identity element of the hyperbolic space loop. Summarizing our discussion we obtain

Theorem 5. There is only one isotopism class $\mathcal{C}$ of the 3 -dimensional connected differentiable Bol loops $L$ such that the group $G$ generated by the left 
translations $\left\{\lambda_{x} ; x \in L\right\}$ is a semisimple Lie group. The group $G$ is isomorphic to $P S L_{2}(\mathbb{C})$ and the stabilizer $H$ of $e \in L$ in $G$ is isomorphic to $S_{3}(\mathbb{R})$. Any loop $L_{a} \quad(-1<a<1)$ in this class $\mathcal{C}$ is isotopic to the hyperbolic space loop $L_{0}$. The tangent space $T_{1} \Lambda$ of the set $\Lambda$ of the left translations of the hyperbolic space loop $L_{0}$ at the identity $1 \in G$ is the plane $\mathbf{m}_{\mathbf{0}}$ through 0 in the Lie algebra $\mathbf{g}$ of $G$ such that $\mathbf{m}_{0}$ is orthogonal to the 3-dimensional Lie algebra $\mathbf{h}$ of $H$ with respect to the Cartan-Killing form $k_{\mathbb{R}}$ of $\mathbf{g}$.

In the class $\mathcal{C}$ only the hyperbolic space loop is a Bruck loop.

The tangential spaces $T_{1} \sigma_{a}(G / H)$ at $1 \in G$ for all $a \in(-1,1)$ are the Bol complements $\mathbf{m}_{a}$. The loops $L_{a}$ and $L_{b}$ in $\mathcal{C}$ are isomorphic if and only if the angles between the 3-spaces $\mathbf{m}_{a}$ and $\mathbf{m}_{0}$, respectively $\mathbf{m}_{b}$ and $\mathbf{m}_{0}$ are the same with respect to $k_{\mathbb{R}}$. As representatives of the isomorphism classes of the loops belonging to $\mathcal{C}$ we may choose the loops $L_{a}$, where $0 \leq a<1$.

\section{3-dimensional Bol loops corresponding to 4-dimensional non-solvable Lie groups}

In this section we determine all 3-dimensional connected differentiable global Bol loops $L$ having a 4-dimensional non-solvable Lie group $G$ as the group topologically generated by their left translations.

Since according to Proposition 2 b) a group locally isomorphic to $S O_{3}(\mathbb{R}) \ltimes \mathbb{R}$ cannot be the group topologically generated by the left translations of a 3 dimensional Bol loop we have to consider only the case that $G$ is locally isomorphic to $P S L_{2}(\mathbb{R}) \times \mathbb{R}$. Moreover, since the group $G$ is isomorphic to the Lie group $G^{*}$ described in $\mathbf{5 . 2}$ and $\mathbf{5 . 3}$ of section 3 we have to discuss the following situation.

Using the $\mathbb{R}$-basis B2 (5.2, Section 3) the 1-dimensional subalgebras $\mathbf{h}$ of $\mathbf{g}=s l_{2}(\mathbb{R}) \times \mathbb{R}$ containing no non-zero ideal of $\mathbf{g}$ are the following:

$\mathbf{h}_{1}=\left\langle e_{2}+k e_{1}\right\rangle$

$\mathbf{h}_{2}=\left\langle\left(e_{3}+e_{4}\right)+k e_{1}\right\rangle$

$\mathbf{h}_{3}=\left\langle e_{4}+k e_{1}\right\rangle$, where $k \in \mathbb{R}$.

Using the automorphism $A: \mathbf{g} \rightarrow \mathbf{g}$ given by $A\left(e_{1}\right)=k e_{1}, A\left(e_{i}\right)=e_{i}$ for $2 \leq i \leq 4$ we may assume that $k=0,1$.

Since the Lie triple system $\mathbf{m}_{1}$ in the case $\mathbf{5 . 2}$ is generated by the basis elements $e_{1}, e_{2}, e_{4}$, the Lie algebras $\mathbf{h}_{1}$ and $\mathbf{h}_{3}$ cannot be complements to $\mathbf{m}_{1}$ in $\mathbf{g}$. Since the elements $e_{2}+e_{4} \in \mathbf{m}$ and $e_{3}+e_{4} \in \mathbf{h}$ are both parabolic in $s l_{2}(\mathbb{R})$, we have a contradiction to Lemma 3 .

Now we consider the Lie triple system $\mathbf{m}_{2}=\left\langle e_{1}, e_{2}, e_{3}\right\rangle$ in the case $\mathbf{5 . 3}$. The intersection of $\mathbf{h}_{1}$ and $\mathbf{m}_{2}$ is not trivial, therefore $\mathbf{m}_{2}$ is not a complement to 
$\mathbf{h}_{1}$ in $\mathbf{g}$. Since $\mathbf{h}_{2} \cap \mathbf{m}_{2}=\mathbf{h}_{3} \cap \mathbf{m}_{2}=\{0\}$ and there is no element of $\mathbf{m}_{2}$ which is conjugate to an element of $\mathbf{h}_{i}$ for $i=2,3$ we have to deal with both cases. For $k=0$ we have $H_{2}=\exp \mathbf{h}_{2}=\left\{\left(\left(\begin{array}{ll}1 & b \\ 0 & 1\end{array}\right), 1\right), b \in \mathbb{R}\right\}$ and $H_{3}=\exp \mathbf{h}_{3}=\left\{(x, 1), x \in S O_{2}(\mathbb{R})\right\}$. Now $G$ and $H$ are direct products $G=G_{1} \times G_{2}, H=H_{1} \times H_{2}$ with $H_{i} \subset G_{i}(i=1,2)$. The Bol loop $L$ corresponding to a such pair is the direct product of a 2-dimensional Bol loop $L_{1}$ and a 1-dimensional Lie group $L_{2}$ such that the group topologically generated by the left translations of the 2-dimensional Bol loop $L_{1}$ is isomorphic to $P S L_{2}(\mathbb{R})$. Then $G=P S L_{2}(\mathbb{R}) \times G_{2}$, where $G_{2}$ is either $\mathbb{R}$ or $S O_{2}(\mathbb{R})$, and $H=H_{3}$. Moreover $L_{1}$ is isotopic to the hyperbolic plane loop.

For $k=1$ one has $G=P S L_{2}(\mathbb{R}) \times \mathbb{R}, H_{2}=\exp \mathbf{h}_{2}=\left\{\left(\left(\begin{array}{ll}1 & b \\ 0 & 1\end{array}\right), b\right)\right\}$, where $b \in \mathbb{R}$ and $\exp \mathbf{m}=\left\{\left(\left(\begin{array}{cc}x+y & z \\ z & x-y\end{array}\right), k\right)\right\}$ with $x, y, z, k \in \mathbb{R}$, and $x^{2}-y^{2}-z^{2}=1([6]$, p. 24). If for a section $\sigma: G / H \rightarrow G$ the image $\sigma(G / H)$ contains exp $\mathbf{m}$ then any $\operatorname{coset}\left(\left(\begin{array}{cc}1+c & 1 \\ c & 1\end{array}\right), 0\right) H, c \in \mathbb{R}$ should contain precisely one element $s$ of $\sigma(G / H)$. If $c \neq-1$ then $s=$ $\left(\left(\begin{array}{cc}1+c & c \\ c & \frac{c^{2}+1}{1+c}\end{array}\right), 0\right)$. Since $\sigma: G / H \rightarrow G$ is continuous we have $\sigma\left(\left(\left(\begin{array}{cc}0 & 1 \\ -1 & 1\end{array}\right), 0\right) H\right)=\lim _{c \rightarrow-1} \sigma\left(\left(\left(\begin{array}{cc}1+c & 1 \\ c & 1\end{array}\right), 0\right) H\right)=$ $\lim _{c \rightarrow-1}\left(\left(\begin{array}{cc}1+c & c \\ c & \frac{c^{2}+1}{1+c}\end{array}\right), 0\right)$, which is a contradiction. This means that there is no global Bol loop corresponding to the triple $\left(G, H_{2}\right.$, exp m $)$. The other case is $G=P S L_{2}(\mathbb{R}) \times S O_{2}(\mathbb{R}), H_{3}=\left\{(x, x) \mid x \in S O_{2}(\mathbb{R})\right\}$ and $\exp \mathbf{m}=\exp \left\{\left(\lambda_{2} e_{2}+\lambda_{3} e_{3}\right), \lambda_{2}, \lambda_{3} \in \mathbb{R}\right\} \times \exp \left\{\left(\lambda_{1} e_{1}\right), \lambda_{1} \in \mathbb{R}\right\}=M \times S O_{2}(\mathbb{R})$, such that $M$ is the image of the section $\sigma_{1}$ given by

$$
\left(\begin{array}{cc}
a & 0 \\
b & a^{-1}
\end{array}\right) \mapsto\left(\begin{array}{cc}
a & 0 \\
b & a^{-1}
\end{array}\right)\left(\begin{array}{cc}
\frac{a^{-1}+a}{ \pm \sqrt{b^{2}+\left(a^{-1}+a\right)^{2}}} & \frac{b}{ \pm \sqrt{b^{2}+\left(a^{-1}+a\right)^{2}}} \\
-\frac{b}{ \pm \sqrt{b^{2}+\left(a^{-1}+a\right)^{2}}} & \frac{a^{-1}+a}{ \pm \sqrt{b^{2}+\left(a^{-1}+a\right)^{2}}}
\end{array}\right)
$$

$\operatorname{choosing} \operatorname{sign}\left( \pm \sqrt{b^{2}+\left(a^{-1}+a\right)^{2}}\right)=\operatorname{sign} b$ if $b \neq 0$ and +1 for $b=0$. The section $\sigma_{1}$ corresponds to the hyperbolic plane loop (cf. [18], pp. 283-284). Since $[[\mathbf{m}, \mathbf{m}], \mathbf{m}] \subseteq \mathbf{m}$ and each element $g \in G$ can uniquely be represented as a product $g=m h$ with $m \in \exp \mathbf{m}$ and $h \in H_{3}$ we have a global Bol loop $L$ defined on the factor space $G / H_{3}$ (cf. 9, Corollary 3.11, p. 51 and [18], Lemma 1.3 , p. 17 ). In $L$ there is a normal subgroup $\tilde{G}$ isomorphic 
to $S_{2}(\mathbb{R})$ and the factor loop $L / \tilde{G}$ is isomorphic to the hyperbolic plane loop. Therefore is $L$ the unique Scheerer extension of the Lie group $S_{2}(\mathbb{R})$ by the hyperbolic plane loop (cf. [18], Section 2). This loop is a left A-loop, because of $[\mathbf{h}, \mathbf{m}] \subseteq \mathbf{m}$. But it is not a Bruck loop since there is no involutory automorphism $\sigma: \mathbf{g} \rightarrow \mathbf{g}$ such that $\sigma(\mathbf{m})=-\mathbf{m}$ and $\sigma(\mathbf{h})=\mathbf{h}$.

Theorem 6. There are precisely three isotopism classes $\mathcal{C}_{1}, \mathcal{C}_{2}, \mathcal{C}_{3}$ of connected differentiable Bol loops $L$ with dimension 3 such that the group $G$ generated by the left translations $\left\{\lambda_{x} ; x \in L\right\}$ is a 4-dimensional non-solvable Lie group. Every loop in the class $\mathcal{C}_{1}$, respectively $\mathcal{C}_{2}$ is the direct product of a 2-dimensional loop isotopic to the hyperbolic plane loop with the Lie group $\mathbb{R}$, respectively $S_{2}(\mathbb{R})$. In the class $\mathcal{C}_{3}$ is contained up to isomorphisms only the Scheerer extension of the Lie group $\mathrm{SO}_{2}(\mathbb{R})$ by the hyperbolic plane loop.

\section{3-dimensional Bol loops belonging to 5-dimensional non-solvable Lie groups}

In this section we seek for 3-dimensional connected differentiable global Bol loops having a 5-dimensional non-solvable Lie group $G$ as the group topologically generated by their left translations.

Any 5-dimensional non-solvable Lie group is locally isomorphic to one of the following groups:

1) $P S L_{2}(\mathbb{R}) \times \mathbb{R}^{2}$

2) $P S L_{2}(\mathbb{R}) \times \mathcal{L}_{2}$, where $\mathcal{L}_{2} \cong\{x \mapsto a x+b ; a>0, b \in \mathbb{R}\}$.

3) $\mathrm{SO}_{3}(\mathbb{R}) \times \mathbb{R}^{2}$

4) $\mathrm{SO}_{3}(\mathbb{R}) \times \mathcal{L}_{2}$

5) The semidirect product $G=S L_{2}(\mathbb{R}) \ltimes \mathbb{R}^{2}$ such that $S L_{2}(\mathbb{R})$ acts in the natural way on $\mathbb{R}^{2}$. The group $G$ may be seen as the connected component of the group of area preserving affinities of the real plane.

Since there is no epimorphism from a 6-dimensional Lie algebra $\mathbf{g}^{*}$ given in section 3 onto one of the 5 -dimensional Lie algebras $\mathbf{g}$ listed above only the Lie algebra $\mathbf{g}=s l_{2}(\mathbb{R}) \ltimes \mathbb{R}^{2}$, which is the Lie algebra of the isometry group of a symmetric space (see case $\mathbf{7}$ in section 3 ) can occur as the group topologically generated by the left translations of a 3 -dimensional connected differentiable Bol loop. Every 2-dimensional connected subgroup $H$ of $G$, which does not have any non-trivial normal subgroup of $G$, contains 1-parameter subgroups $S$ fixing a point or contains a 1-dimensional translation group. The Lie algebra of $S$ has as generator an elliptic, parabolic, or hyperbolic element of $\mathbf{g}$. Using the relations of $\mathbf{7}$ we see that $\mathbf{m}$ contains elements corresponding to translations and $\mathbf{m} \cap s_{2}(\mathbb{R})$ has with $e_{2}$ a hyperbolic, with $e_{3}$ an elliptic and 
with $e_{2}+e_{3}$ a parabolic element. Therefore exp $\mathbf{m}$ contains elements conjugate to elements of $H$. According to Lemma 3 there is no 3-dimensional Bol loop $L$ as section in the group $P S L_{2}(\mathbb{R}) \ltimes \mathbb{R}^{2}$. This consideration yields the following

Theorem 7. There is no 3-dimensional connected differentiable global Bol loop L having a 5-dimensional non-solvable Lie group as the group topologically generated by its left translations.

\section{3-dimensional Bol loops with 6-dimensional non-solvable Lie groups as their left trans- lations groups}

A 6-dimensional non-solvable Lie group $G$ can be occur as the group topologically generated by the left translations of a 3 -dimensional connected differentiable Bol loop, if $G$ is isomorphic to a 6-dimensional Lie group $G^{*}$ listed in the section 3. Then we have to deal with the cases, where $G$ is locally isomorphic to one of the following Lie groups:

1) The connected component of the euclidean motion group on $\mathbb{R}^{3}$.

2) The semidirect product of $P S L_{2}(\mathbb{R}) \ltimes \mathbb{R}^{3}$, where the action of $P S L_{2}(\mathbb{R})$ on $\mathbb{R}^{3}$ is the adjoint action of $P S L_{2}(\mathbb{R})$ on its Lie algebra.

Let us consider the first case. The 3-dimensional subgroups $H$ of $G=$ $S_{3}(\mathbb{R}) \ltimes \mathbb{R}^{3}$, which does not contain any non-trivial normal subgroup of $G$, are locally isomorphic to the following subgroups: Either $H$ has the shape as in case c) of Proposition 2 and there is no 3-dimensional differentiable Bol loop corresponding to the pair $(G, H)$ or $H=\left\{(a, 0), a \in S O_{3}(\mathbb{R})\right\}$. We consider the $\mathbb{R}$-basis B3 of the Lie algebra $\mathrm{g}=\mathrm{so}_{3}(\mathbb{R}) \ltimes \mathbb{R}^{3}$ given in the case 6.1 (Section 3). Then the Lie algebra $\mathbf{h}$ of $H$ is generated by the basis elements $e_{2}, e_{3}, e_{4}$ and the Lie triple system $\mathbf{m}$ is generated by the basis elements $e_{1}, e_{2}, e_{3}$. Then $\mathbf{h} \cap \mathbf{m}=\left\langle e_{2}, e_{3}\right\rangle$ which is a contradiction.

Now we discuss the second case. Then the group multiplication is given by

$$
\left(A_{1}, X_{1}\right) \circ\left(A_{2}, X_{2}\right)=\left(A_{1} A_{2}, A_{2}^{-1} X_{1} A_{2}+X_{2}\right),
$$

where $\left(A_{i}, X_{i}\right), i=1,2$ are two elements of $G$ such that $X_{i} i=1,2$ are represented by $2 \times 2$ real matrices with trace 0 . We use the $\mathbb{R}$-basis B4 of the Lie algebra $\mathbf{g}=s l_{2}(\mathbb{R}) \ltimes \mathbb{R}^{3}$ (6.3, Section 3$)$. The 3-dimensional subalgebras $\mathbf{h}$ of $\mathbf{g}$ containing no non-zero ideal of $\mathbf{g}$ are the following:

a) $\left\langle e_{2}, e_{5}, e_{1}+e_{6}\right\rangle$, 
b) $\left\langle e_{2}+k e_{5}, e_{1}, e_{6}\right\rangle$, where $k \in \mathbb{R}$,

c) $\left\langle e_{3}+e_{4}, e_{5}, e_{1}-e_{6}\right\rangle$,

d) $\left\langle e_{2}, e_{3}+e_{4}, e_{1}-e_{6}\right\rangle$,

e) $\left\langle e_{2}, e_{3}, e_{4}\right\rangle$,

f) $\left\langle e_{4}, e_{5}, e_{6}\right\rangle$.

First we deal with the Lie triple system $\mathbf{m}$ of (6.2 in section 3) generated by the basis elements $e_{4}, e_{2}, e_{6}$. If the Lie algebra $\mathbf{h}$ has the shape a), b), d), e) and $\mathbf{f}$ ) then the intersection of $\mathbf{h}$ with $\mathbf{m}$ are the subspaces $\left\langle e_{2}\right\rangle,\left\langle e_{6}\right\rangle,\left\langle e_{2}\right\rangle$, $\left\langle e_{2}, e_{4}\right\rangle$, or $\left\langle e_{4}, e_{6}\right\rangle$ respectively. In the case c) the elements $e_{2}+e_{4} \in \mathbf{m}$ and $e_{3}+e_{4} \in \mathbf{h}$ are parabolic and the parabolic elements of $s l_{2}(\mathbb{R})$ are conjugate to each other. This is a contradiction to Lemma 3.

Let us treat the Lie triple system $\mathbf{m}$ of (6.3 in section 3$)$ which is generated by the basis elements $e_{1}, e_{2}, e_{3}$ and the image of $\mathbf{m}$ under the exponential map.

The exponential map exp : $\mathbf{g} \rightarrow G$ is defined in the following way: For $X \in \mathrm{g}$ we have exp $X=\gamma_{X}(1)$, where $\gamma_{X}(t)$ is the 1-parameter subgroup of $G$ with the property $\left.\frac{d}{d t}\right|_{t=0} \gamma_{X}(t)=X$. In the 1-parameter subgroup $\alpha(t)=$ $(\beta(t), \gamma(t))$ of $G$ with the conditions $\alpha(t=0)=(1,0)$ and $\left.\frac{d}{d t}\right|_{t=0} \alpha(t)=$ $\left(X_{1}, X_{2}\right)=X \in \mathbf{g}$ the first component $\beta(t)$ is the 1-parameter subgroup of $P S L_{2}(\mathbb{R})$, and the second component satisfies

$\frac{d}{d t} \gamma(t)=\left.\frac{d}{d s}\right|_{s=0} \gamma(t+s)=-\left.\frac{d}{d s}\right|_{s=0} \beta(s) \gamma(t)+\left.\gamma(t) \frac{d}{d s}\right|_{s=0} \beta(s)+\left.\frac{d}{d s}\right|_{s=0} \gamma(s)=$ $-X_{1} \gamma(t)+\gamma(t) X_{1}+X_{2}$.

For $X_{1}=\left(\begin{array}{rr}a & b \\ c & -a\end{array}\right)$, and $X_{2}=\left(\begin{array}{rr}k & u \\ y & -k\end{array}\right)$, with $a, b, c, k, u, y \in \mathbb{R}$ and $\gamma(t)=\left(\begin{array}{rr}r(t) & s(t) \\ v(t) & -r(t)\end{array}\right)$, one has

$$
\begin{aligned}
\frac{d}{d t} \gamma(t)= & \left(\begin{array}{rr}
\frac{d}{d t} r(t) & \frac{d}{d t} s(t) \\
\frac{d}{d t} v(t) & -\frac{d}{d t} r(t)
\end{array}\right)=\left(\begin{array}{rr}
-a & -b \\
-c & a
\end{array}\right)\left(\begin{array}{rr}
r(t) & s(t) \\
v(t) & -r(t)
\end{array}\right)+ \\
& \left(\begin{array}{rr}
r(t) & s(t) \\
v(t) & -r(t)
\end{array}\right)\left(\begin{array}{rr}
a & b \\
c & -a
\end{array}\right)+\left(\begin{array}{rr}
k & u \\
y & -k
\end{array}\right)
\end{aligned}
$$

with the following properties:

$$
\begin{gathered}
r(0)=s(0)=v(0)=0,\left.\frac{d}{d t}\right|_{t=0} r(t)=k,\left.\frac{d}{d t}\right|_{t=0} s(t)=u,\left.\frac{d}{d t}\right|_{t=0} v(t)=y, \\
\frac{d}{d t} r(t)=-b v(t)+c s(t)+k, \frac{d}{d t} s(t)=2(b r(t)+a s(t))+u
\end{gathered}
$$




$$
\frac{d}{d t} v(t)=2(a v(t)-c r(t))+y
$$

The solution of this inhomogeneous system of linear differential equations is:

$$
\begin{gathered}
r(t)=\frac{1}{8\left(a^{2}+b c\right)^{\frac{3}{2}}}\left(e^{2 \sqrt{a^{2}+b c} t}-e^{-2 \sqrt{a^{2}+b c} t}\right)(-a c u-b a y+2 k c b)+ \\
\frac{1}{8\left(a^{2}+b c\right)}\left[\left(e^{\sqrt{a^{2}+b c} t}-e^{-\sqrt{a^{2}+b c} t}\right)^{2}(-c u+b y)+t\left(8 k a^{2}+4 a c u+4 a b y\right)\right], \\
s(t)=\frac{1}{8\left(a^{2}+b c\right)^{\frac{3}{2}}}\left(e^{2 \sqrt{a^{2}+b c} t}-e^{-2 \sqrt{a^{2}+b c} t}\right)\left(-b^{2} y+u b c-2 b a k+2 a^{2} u\right)+ \\
\frac{1}{8\left(a^{2}+b c\right)}\left[\left(e^{\sqrt{a^{2}+b c} t}-e^{-\sqrt{a^{2}+b c} t}\right)^{2}(-2 b k+2 a u)+t\left(4 b^{2} y+4 u b c+8 k a b\right)\right], \\
v(t)=\frac{1}{8\left(a^{2}+b c\right)^{\frac{3}{2}}}\left(e^{2 \sqrt{a^{2}+b c} t}-e^{-2 \sqrt{a^{2}+b c} t}\right)\left(2 y a^{2}-2 c k a+b c y-c^{2} u\right)+ \\
\frac{1}{8\left(a^{2}+b c\right)}\left[\left(e^{\sqrt{a^{2}+b c} t}-e^{-\sqrt{a^{2}+b c} t}\right)^{2}(-2 a y+2 c k)+t\left(8 c k a+4 c^{2} u+4 b c y\right)\right] .
\end{gathered}
$$

It follows exp $\mathbf{m}=\left\{\exp \left(X_{1}, X_{2}\right)\right\}$, where $X_{1} \in\left\{\left(\begin{array}{cc}\lambda_{2} & \lambda_{3} \\ \lambda_{3} & -\lambda_{2}\end{array}\right), \lambda_{2}, \lambda_{3} \in \mathbb{R}\right\}$ and $X_{2} \in\left\{\left(\begin{array}{cc}0 & -\lambda_{1} \\ \lambda_{1} & 0\end{array}\right), \lambda_{1} \in \mathbb{R}\right\}$.

The first component of $\exp \mathbf{m}$ is

$$
\left(\exp \left(X_{1}, X_{2}\right)\right)_{1}=\left(\begin{array}{cc}
\cosh \sqrt{A}+\frac{\sinh \sqrt{A}}{\sqrt{A}} \lambda_{2} & \frac{\sinh \sqrt{A}}{\sqrt{A}} \lambda_{3} \\
\frac{\sinh \sqrt{A}}{\sqrt{A}} \lambda_{3} & \cosh \sqrt{A}-\frac{\sinh \sqrt{A}}{\sqrt{A}} \lambda_{2}
\end{array}\right)
$$

where $A=\lambda_{2}^{2}+\lambda_{3}^{2}$ (cf. [6], p. 24); the second component of $\exp \mathbf{m}$ is $\left(\exp \left(X_{1}, X_{2}\right)\right)_{2}=\left(\begin{array}{rr}r(1) & s(1) \\ v(1) & -r(1)\end{array}\right)$, where

$$
\begin{gathered}
r(1)=\frac{\lambda_{3} \lambda_{1}}{4\left(\lambda_{2}^{2}+\lambda_{3}^{2}\right)}\left(e^{\sqrt{\lambda_{2}^{2}+\lambda_{3}^{2}}}-e^{-\sqrt{\lambda_{2}^{2}+\lambda_{3}^{2}}}\right)^{2}, \\
s(1)=\frac{-\lambda_{1}}{4 \sqrt{\lambda_{2}^{2}+\lambda_{3}^{2}}}\left(e^{2 \sqrt{\lambda_{2}^{2}+\lambda_{3}^{2}}}-e^{-2 \sqrt{\lambda_{2}^{2}+\lambda_{3}^{2}}}\right)-\frac{\lambda_{2} \lambda_{1}}{4\left(\lambda_{2}^{2}+\lambda_{3}^{2}\right)}\left(e^{\sqrt{\lambda_{2}^{2}+\lambda_{3}^{2}}}-e^{-\sqrt{\lambda_{2}^{2}+\lambda_{3}^{2}}}\right)^{2}, \\
v(1)=\frac{\lambda_{1}}{4 \sqrt{\lambda_{2}^{2}+\lambda_{3}^{2}}}\left(e^{2 \sqrt{\lambda_{2}^{2}+\lambda_{3}^{2}}}-e^{-2 \sqrt{\lambda_{2}^{2}+\lambda_{3}^{2}}}\right)-\frac{\lambda_{2} \lambda_{1}}{4\left(\lambda_{2}^{2}+\lambda_{3}^{2}\right)}\left(e^{\sqrt{\lambda_{2}^{2}+\lambda_{3}^{2}}}-e^{-\sqrt{\lambda_{2}^{2}+\lambda_{3}^{2}}}\right)^{2} .
\end{gathered}
$$


The Lie algebra $\mathbf{h}$ of the stabilizer cannot have the shape a), b), d) and e) since in these cases the intersection of $\mathbf{h}$ with $\mathbf{m}$ is the subspace $\left\langle e_{2}\right\rangle,\left\langle e_{1}\right\rangle$, $\left\langle e_{2}\right\rangle$ and $\left\langle e_{2}, e_{3}\right\rangle$ respectively.

In the case c) we have $\mathbf{h} \cap \mathbf{m}=0$, there is no element of $\mathbf{m}$ which is conjugate to an element of $\mathbf{h}$ and

$$
H=\left\{\left(\left(\begin{array}{ll}
1 & b \\
0 & 1
\end{array}\right),\left(\begin{array}{cc}
e & f \\
0 & -e
\end{array}\right)\right) ; b, e, f \in \mathbb{R}\right\} .
$$

To prove that in this case there is no global Bol loop we may proceed as we did it for the group $G=P S L_{2}(\mathbb{R}) \times \mathbb{R}$ and for the stabilizer

$\exp \mathbf{h}_{2}=\left\{\left(\left(\begin{array}{ll}1 & b \\ 0 & 1\end{array}\right), b\right) ; b \in \mathbb{R}\right\}$ since the first component of $H$ is equal to the first component of $\exp \mathbf{h}_{2}$ and the first components of $\exp \mathbf{m}$ are the same in both cases.

Now we assume that the stabilizer $H$ has the shape f). Since the group $S L_{2}(\mathbb{R})$ has no 3-dimensional linear representation the group $G$ is isomorphic to the semidirect product of $P S L_{2}(\mathbb{R}) \ltimes \mathbb{R}^{3}$ and $H$ may be chosen as $H=\left\{\left( \pm\left(\begin{array}{rr}\cos t & \sin t \\ -\sin t & \cos t\end{array}\right),\left(\begin{array}{rr}-x & y \\ y & x\end{array}\right)\right), t \in[0,2 \pi), x, y \in \mathbb{R}\right\}$.

We have $[\mathbf{m}, \mathbf{m}]=\mathbf{h}, \mathbf{g}=\mathbf{m} \oplus[\mathbf{m}, \mathbf{m}]$ and therefore the corresponding loop $L$ realized on $G / H$ is a Bruck loop. We prove that this loop is global.

For the connected simple Lie group $P S L_{2}(\mathbb{R})$ there exists a unique decomposition $g=\left(\begin{array}{cc}a & 0 \\ b & a^{-1}\end{array}\right)\left( \pm\left(\begin{array}{rr}\cos t & \sin t \\ -\sin t & \cos t\end{array}\right)\right)$, where $a>0, b \in \mathbb{R}$, $t \in[0,2 \pi)($ cf. [7], p. 525). Since

$$
\begin{gathered}
\left( \pm\left(\begin{array}{rr}
\cos t & -\sin t \\
\sin t & \cos t
\end{array}\right)\right)\left(\begin{array}{rr}
0 & u \\
-u & 0
\end{array}\right)\left( \pm\left(\begin{array}{rr}
\cos t & \sin t \\
-\sin t & \cos t
\end{array}\right)\right)+\left(\begin{array}{rr}
k & l \\
l & -k
\end{array}\right)= \\
\left(\begin{array}{rr}
0 & u \\
-u & 0
\end{array}\right)+\left(\begin{array}{rr}
k & l \\
l & -k
\end{array}\right)=\left(\begin{array}{cc}
k & u+l \\
l-u & -k
\end{array}\right)
\end{gathered}
$$

each element of $G$ can uniquely be written as $\left( \pm\left(\begin{array}{ll}a & b \\ c & d\end{array}\right),\left(\begin{array}{rr}x & y \\ z & -x\end{array}\right)\right)=$ $\left(\left(\begin{array}{ll}a_{1} & 0 \\ b_{1} & a_{1}^{-1}\end{array}\right),\left(\begin{array}{rr}0 & u \\ -u & 0\end{array}\right)\right) \circ\left( \pm\left(\begin{array}{rr}\cos t & \sin t \\ -\sin t & \cos t\end{array}\right),\left(\begin{array}{rr}k & l \\ l & -k\end{array}\right)\right)$ with $a, b, c, d \in \mathbb{R}, a d-b c=1, x, y, z \in \mathbb{R}, a_{1}>0, b_{1}, u, k, l \in \mathbb{R}, t \in[0,2 \pi)$, such that $k=x, l=\frac{y+z}{2}, u=\frac{y-z}{2}$. The submanifold exp $\mathbf{m}$ is the image of a section $\tau: G / H \rightarrow G$ if and only if each element $g \in G$ can uniquely be represented as a product $g=m h$ with $m \in \exp \mathbf{m}$ and $h \in H$ or equivalently $m=g h^{-1}$. It is sufficient to prove this for each element $g \in G$ with the 
shape

$$
\left\{\left(\left(\begin{array}{ll}
a & 0 \\
b & a^{-1}
\end{array}\right),\left(\begin{array}{rr}
0 & u \\
-u & 0
\end{array}\right)\right), a>0, b, u \in \mathbb{R}\right\} .
$$

The first component of $\exp \mathbf{m}$ is precisely the section $\sigma_{1}$ of the hyperbolic plane loop given before Theorem 6 . Therefore for given $a>0, b \in \mathbb{R}$ we have unique $\lambda_{2}, \lambda_{3} \in \mathbb{R}, t \in[0,2 \pi)$ such that

$$
\begin{gathered}
\left(\begin{array}{cc}
\cosh \sqrt{A}+\frac{\sinh \sqrt{A}}{\sqrt{A}} \lambda_{2} & \frac{\sinh \sqrt{A}}{\sqrt{A}} \lambda_{3} \\
\frac{\sinh \sqrt{A}}{\sqrt{A}} \lambda_{3} & \cosh \sqrt{A}-\frac{\sinh \sqrt{A}}{\sqrt{A}} \lambda_{2}
\end{array}\right)= \\
\left(\begin{array}{ll}
a & 0 \\
b & a^{-1}
\end{array}\right)\left(\begin{array}{rr}
\cos t & \sin t \\
-\sin t & \cos t
\end{array}\right),
\end{gathered}
$$

where $A=\lambda_{2}^{2}+\lambda_{3}^{2}$. Hence we have to consider the second component of $\exp \mathbf{m}$. For given $u, \lambda_{2}, \lambda_{3}$ we have to find unique $\lambda_{1}, k, l \in \mathbb{R}$ such that

$$
\left(\begin{array}{rr}
r(1) & s(1) \\
v(1) & -r(1)
\end{array}\right)=\left(\begin{array}{cc}
k & l+u \\
l-u & -k
\end{array}\right)
$$

where $r(1), s(1), v(1)$ are values of functions, which depend on the variables $\lambda_{1}, \lambda_{2}, \lambda_{3}$. Since for $\lambda_{1}$ we obtain the equation

$$
2 u=\frac{-\lambda_{1}}{2 \sqrt{\lambda_{2}^{2}+\lambda_{3}^{2}}}\left(e^{2 \sqrt{\lambda_{2}^{2}+\lambda_{3}^{2}}}-e^{-2 \sqrt{\lambda_{2}^{2}+\lambda_{3}^{2}}}\right)
$$

we have the unique solutions $\lambda_{1}=\frac{-4 u \sqrt{\lambda_{2}^{2}+\lambda_{3}^{2}}}{e^{2 \sqrt{\lambda_{2}^{2}+\lambda_{3}^{2}}}-e^{-2 \sqrt{\lambda_{2}^{2}+\lambda_{3}^{2}}}}, k=r(1)$ and $l=\frac{s(1)+v(1)}{2}$.

Summarizing the above discussion we see that $\exp \mathbf{m}$ is an image of a global section $\sigma: G / H \rightarrow G$. The section $\sigma$ determines a global Bol loop $(\hat{L}, *)$ since $[[\mathbf{m}, \mathbf{m}], \mathbf{m}] \subseteq \mathbf{m}$ and therefore the equation $x * a=b$ has the solution $x=a^{-1} *\left[(a * b) * a^{-1}\right]$ for all $a, b \in \hat{L}$ (cf. [9], Corollary 3.11, p. 51 and [18], Lemma 1.3 , p. 17$)$. We call the loop $(\hat{L}, *)$ the pseudo-euclidean space loop.

In order to determine within the isotopism class $\mathcal{C}$ of the pseudo-euclidean space loop $(\hat{L}, *)$ the isomorphism classes we consider arbitrary complements $\mathbf{m}$ to the Lie algebra $\mathbf{h}$ of the stabilizer $H$ of $e \in L$ in $\mathbf{g}$. These complements have the form:

$$
\left\langle e_{1}+a_{1} e_{4}+a_{2} e_{5}+a_{3} e_{6}, e_{2}+b_{1} e_{4}+b_{2} e_{5}+b_{3} e_{6}, e_{3}+c_{1} e_{4}+c_{2} e_{5}+c_{3} e_{6}\right\rangle
$$


where $a_{1}, a_{2}, a_{3}, b_{1}, b_{2}, b_{3}, c_{1}, c_{2}, c_{3} \in \mathbb{R}$. Such complements are Bol complements if and only if

$$
\mathbf{m}=\mathbf{m}_{b_{3}, c_{3}, c_{2}}=\left\langle e_{1}-c_{3} e_{6}+b_{3} e_{5}, e_{2}+c_{2} e_{6}+b_{3} e_{4}, e_{3}+c_{2} e_{5}+c_{3} e_{4}\right\rangle,
$$

where $c_{2}, c_{3}, b_{3} \in \mathbb{R}$, and $b_{3}^{2}+c_{3}^{2} \neq 1$. According to remark in section 2 the subspace $\mathbf{m}_{b_{3}, c_{3}, c_{2}}$ belongs to a loop within the isotopism class $\mathcal{C}$ if and only if the subalgebra $\mathbf{h}_{b_{3}, c_{3}, c_{2}}=\left[\mathbf{m}_{b_{3}, c_{3}, c_{2}}, \mathbf{m}_{b_{3}, c_{3}, c_{2}}\right]$ is the Lie algebra of the semidirect product of a 2-dimensional normal translation group $T$ by a 1 dimensional rotation group $S$. It follows that then $\mathbf{h}_{b_{3}, c_{3}, c_{2}}=\mathbf{t} \oplus \mathbf{s}$, where $\mathbf{t}$ respectively $\mathbf{s}$ is the Lie algebra of $T$ respectively of $S$ and the CartanKilling form $k$ given by $\mathbf{K} \mathbf{2}$ in section 3 is zero on $\mathbf{t}$ and negative on $\mathbf{s}$. Since $\left[\mathbf{m}_{b_{3}, c_{3}, c_{2}}, \mathbf{m}_{b_{3}, c_{3}, c_{2}}\right]=\left\langle d_{1}, d_{2}, d_{3}\right\rangle$ with

$$
\begin{gathered}
d_{1}=\left(1-b_{3}^{2}\right) e_{6}-c_{3} e_{1}-c_{3} b_{3} e_{5}, d_{2}=\left(1-c_{3}^{2}\right) e_{5}+b_{3} e_{1}-c_{3} b_{3} e_{6}, \\
d_{3}=e_{4}+c_{3} e_{3}+b_{3} e_{2}+c_{2} c_{3} e_{5}+b_{3} c_{2} e_{6},
\end{gathered}
$$

the subalgebra $\left\langle d_{1}, d_{2}\right\rangle$ is an ideal of $\mathbf{h}_{b_{3}, c_{3}, c_{2}}$ and $k\left(d_{3}\right)<0$ if and only if $b_{3}^{2}+c_{3}^{2}<1$.

The Bol complements $\mathbf{m}_{b_{3}, c_{3}, c_{2}}$ with $b_{3}^{2}+c_{3}^{2}>1$ are according to Remark in section 2 symmetric spaces belonging to the Lie triple system given in $\mathbf{6 . 2}$ (section 3). But the local Bol loop corresponding to $\mathbf{g}=\mathbf{m}_{b_{3}, c_{3}, c_{2}} \oplus \mathbf{h}$ cannot be extended to a global Bol loop.

The loop $L$ belonging to $\left(G, H, \exp \mathbf{m}_{b_{3}, c_{3}, c_{2}}\right), b_{3}^{2}+c_{3}^{2}<1$ and the loop $L^{\prime}$ corresponding to $\left(G, H\right.$, exp $\left.\mathbf{m}_{b_{3}^{\prime}, c_{3}^{\prime}, c_{2}^{\prime}}\right), b_{3}^{\prime 2}+c_{3}^{\prime 2}<1$ are isomorphic if and only if there exists an automorphism $\alpha$ of $\mathbf{g}$ such that $\alpha\left(\mathbf{m}_{b_{3}^{\prime}, c_{3}^{\prime}, c_{2}^{\prime}}\right)=\mathbf{m}_{b_{3}, c_{3}, c_{2}}$ and $\alpha(\mathbf{h})=\mathbf{h}$. The automorphism group $A$ of the Lie algebra $\mathbf{g}$ leaving $\mathbf{h}$ invariant consists of linear mappings determined by the basis transformations:

$\alpha\left(e_{1}\right)=a e_{1}$,

$\alpha\left(e_{6}\right)=b_{2} e_{6}+b_{4} e_{5}$,

$\alpha\left(e_{5}\right)=-\varepsilon b_{4} e_{6}+\varepsilon b_{2} e_{5}$,

$\alpha\left(e_{4}\right)=\varepsilon e_{4}+d_{5} e_{6}+d_{6} e_{5}$,

$\alpha\left(e_{2}\right)=\frac{b_{2}}{a} e_{2}+\frac{b_{4}}{a} e_{3}+\left(\frac{-\varepsilon d_{5} b_{4}+\varepsilon d_{6} b_{2}}{a}\right) e_{1}+f_{5} e_{6}+f_{6} e_{5}$,

$\alpha\left(e_{3}\right)=\varepsilon \frac{b_{2}}{a} e_{3}-\varepsilon \frac{b_{4}}{a} e_{2}+\left(\frac{-d_{5} b_{2}-d_{6} b_{4}}{a}\right) e_{1}-\varepsilon f_{6} e_{6}+\varepsilon f_{5} e_{5}$,

where $\varepsilon \in\{1,-1\}$ and for the real parameters $a, b_{2}, b_{4}, d_{5}, d_{6}, f_{5}, f_{6}$ the properties $a^{2}=b_{2}^{2}+b_{4}^{2} \neq 0$ and $b_{2} f_{6}=b_{4} f_{5}$ are satisfied.

According to K2 (Section 3) the Cartan-Killing form $k$ is invariant under $\alpha$. Hence the 3 angles between $\mathbf{m}_{b_{3}, c_{3}, c_{2}}$ and $\hat{\mathbf{m}}:=\mathbf{m}_{0,0,0}$ respectively between $\mathbf{m}_{b_{3}^{\prime}, c_{3}^{\prime}, c_{2}^{\prime}}$ and $\hat{\mathbf{m}}$ are equal with respect to $k$.

Now we compute the isomorphism classes of the loops $L_{b_{3}, c_{3}, c_{2}}$. Since the automorphism $\gamma$ of $\mathbf{g}$ given by $\gamma\left(e_{1}\right)=-e_{1}, \gamma\left(e_{2}\right)=-e_{2}-2 c_{2} e_{4}, \gamma\left(e_{3}\right)=$ $-e_{3}-2 c_{2} e_{5}, \gamma\left(e_{i}\right)=e_{i}$ for $i=4,5,6$ maps $\mathbf{m}_{b_{3}, c_{3}, c_{2}}$ onto $\mathbf{m}_{b_{3}, c_{3}, 0}$ we seek for 
which real triples $\left(b_{3}^{\prime}, c_{3}^{\prime}, 0\right)$ and $\left(b_{3}, c_{3}, 0\right)$ there is an automorphism $\alpha$ in $A$, such that $\alpha\left(\mathbf{m}_{b_{3}^{\prime}, c_{3}^{\prime}, 0}\right)=\mathbf{m}_{b_{3}, c_{3}, 0}$. This condition is equivalent to the following system of equations:

$$
\begin{aligned}
& -a c_{3}=-c_{3}^{\prime} b_{2}-\varepsilon b_{3}^{\prime} b_{4}, \\
& a b_{3}=-c_{3}^{\prime} b_{4}+\varepsilon b_{3}^{\prime} b_{2} \text {, } \\
& a\left(f_{5}+b_{3}^{\prime} d_{5}\right)=\left(\varepsilon d_{5} b_{4}-\varepsilon d_{6} b_{2}\right) c_{3}, \\
& a\left(f_{6}+b_{3}^{\prime} d_{6}\right)=\left(-\varepsilon d_{5} b_{4}+\varepsilon d_{6} b_{2}\right) b_{3}, \\
& a\left(-\varepsilon f_{6}+c_{3}^{\prime} d_{5}\right)=\left(d_{5} b_{2}+d_{6} b_{4}\right) c_{3} \text {, } \\
& a\left(\varepsilon f_{5}+c_{3}^{\prime} d_{6}\right)=\left(-d_{5} b_{2}-d_{6} b_{4}\right) b_{3} .
\end{aligned}
$$

Solving this system of equations we see that the loop $\hat{L}_{d}=L_{d, 0,0}$ for $0 \leq d<1$ is a representative of the isomorphism class consisting of loops $L_{b_{3}, c_{3}, c_{2}}$ with $\sqrt{b_{3}^{2}+c_{3}^{2}}=d$. The pseudo-euclidean space loop $(\hat{L}, *)=L_{0,0,0}$ is a representative of the isomorphism class consisting of the loops $L_{0,0, c_{2}}, c_{2} \in \mathbb{R}$.

A loop isomorphic to $\hat{L}$ is a Bruck loop and hence $\mathbf{m}_{0,0, c_{2}}$ is reductive to $\mathbf{h}$ in $\mathbf{g}$. If $\mathbf{m}_{d, 0,0}$ with $d \neq 0$ were reductive to $\mathbf{h}$ in the Lie algebra $\mathbf{g}$ then for $e_{4} \in \mathbf{h}$ and $e_{1}+d e_{5} \in \mathbf{m}_{d, 0,0}$ the element $\left[e_{4}, e_{1}+d e_{5}\right]=d e_{6} \in \mathbf{h}$ would be contained in $\mathbf{m}_{d, 0,0}$, which is a contradiction.

By $E(2,1)$ we denote the pseudo-euclidean space the points of which are represented by the matrices

$$
(I, Y)=\left(I,\left(\begin{array}{cc}
x & k+l \\
k-l & -x
\end{array}\right)\right)
$$

where $x, k, l \in \mathbb{R}$, and which has as norm $\|(I, Y)\|=x^{2}+k^{2}-l^{2}$. The group $G$ acts on the space $E(2,1)$ in the following way: For given $(A, X) \in G$ and $(I, Y) \in E(2,1)$

$$
(*) \quad(A, X) *(I, Y)=\left(I, A^{-1} Y A+X\right) .
$$

The norm is invariant under the action of $G$, therefore $G$ is the connected component of the motion group of $E(2,1)$.

The 3-dimensional pseudo-euclidean geometry $E(2,1)$ has also a representation $\mathcal{R}$ on the affine space $\mathbb{R}^{3}$ such that the motion group consists of the affine mappings

$$
(B, b):(x, y, z) \mapsto(x, y, z) B^{T}+b,
$$

where $B=\left(\begin{array}{lll}a_{1} & a_{2} & a_{3} \\ b_{1} & b_{2} & b_{3} \\ c_{1} & c_{2} & c_{3}\end{array}\right)$ with $\operatorname{det} B=1, a_{1}^{2}+a_{2}^{2}=a_{3}^{2}+1, b_{1}^{2}+b_{2}^{2}=b_{3}^{2}+1$, $c_{1}^{2}+c_{2}^{2}=c_{3}^{2}-1$, and $b=\left(b_{1}, b_{2}, b_{3}\right)([2]$, Kapitel 6$)$. The mappings

$$
\omega:\left(I,\left(\begin{array}{cc}
k & l+n \\
l-n & -k
\end{array}\right)\right) \mapsto(k, l, n)
$$


and

$$
\begin{aligned}
& \Omega:\left( \pm\left(\begin{array}{ll}
a & b \\
c & d
\end{array}\right),\left(\begin{array}{cc}
x & y+z \\
y-z & -x
\end{array}\right)\right) \mapsto \\
& \left.\left(\begin{array}{ccc}
d a+b c & c d-b a & c d+b a \\
b d-c a & \frac{a^{2}+d^{2}-b^{2}-c^{2}}{2} & \frac{d^{2}+b^{2}-a^{2}-c^{2}}{2} \\
b d+c a & \frac{d^{2}-b^{2}-a^{2}+c^{2}}{2} & \frac{d^{2}+b^{2}+a^{2}+c^{2}}{2}
\end{array}\right),(x, y, z)\right)
\end{aligned}
$$

establish an isometry from $E(2,1)$ onto $\mathcal{R}$ ([10], pp. 97-103).

The stabilizer $H$ which is the image of the Lie algebra of the shape f) under the exponential map leaves in $E(2,1)$ the plane $P$ consisting of the points $\left\{\left(I,\left(\begin{array}{rr}x & y \\ y & -x\end{array}\right)\right), x, y \in \mathbb{R}\right\}$ invariant. The points of $P$ satisfy $x^{2}+y^{2}>0$. The planes of $\mathcal{R}$ the points $(x, y, z)$ of which satisfy $x^{2}+y^{2}>z^{2}$ are called euclidean planes. The connected component $\Omega(G)$ of the motion group of $\mathcal{R}$ acts transitively on the set $\Psi$ of the euclidean planes and the set $\Omega\left(\exp \mathbf{m}_{0,0,0}\right)$ is sharply transitive on $\Psi$. The planes of $\Psi$ can be taken as the points of the pseudo-euclidean space loop $(\hat{L}, *)$ such that the multiplication is given by

$$
\text { (**) } \quad Q_{1} * Q_{2}=\tau_{P, Q_{1}}\left(Q_{2}\right), \quad \text { for all } Q_{1}, Q_{2} \in \Psi
$$

where $\tau_{P, Q_{1}}$ is the unique element of $\Omega\left(\exp \mathbf{m}_{0,0,0}\right)$ mapping the plane $P$, which is the identity of $\hat{L}$ onto $Q_{1}$.

From the above discussion we obtain the

Theorem 8. There is only one isotopism class $\mathcal{C}$ of the 3-dimensional connected differentiable Bol loops $L$ such that the group $G$ generated by the left translations $\left\{\lambda_{x} ; x \in L\right\}$ is a 6-dimensional non-semisimple and non-solvable Lie group. The group $G$ is isomorphic to $P S L_{2}(\mathbb{R}) \ltimes \mathbb{R}^{3}$, where the action of $P S L_{2}(\mathbb{R})$ on $\mathbb{R}^{3}$ is the adjoint action of $P S L_{2}(\mathbb{R})$ on its Lie algebra. The stabilizer $H$ of $e \in L$ in $G$ is isomorphic to

$$
H=\left\{\left( \pm\left(\begin{array}{rr}
\cos t & \sin t \\
-\sin t & \cos t
\end{array}\right),\left(\begin{array}{rr}
-x & y \\
y & x
\end{array}\right)\right) ; t \in[0,2 \pi], x, y \in \mathbb{R}\right\} .
$$

The class $\mathcal{C}$ consists of Bol loops $L_{b_{3}, c_{3}, c_{2}}$, where $b_{3}, c_{3}, c_{2}$ are real parameters and $b_{3}^{2}+c_{3}^{2}<1$. As a representative of $\mathcal{C}$ one may choose the pseudo-euclidean space loop $\hat{L}=L_{0,0,0}$. This loop is a Bruck loop.

The tangential spaces $T_{1} \sigma(G / H)$ of the loops $L_{b_{3}, c_{3}, c_{2}}$ at $1 \in G$ are the Bol complements $\mathbf{m}_{b_{3}, c_{3}, c_{2}}$. The loops $L$ and $L^{\prime}$ in $\mathcal{C}$ are isomorphic if and only if the angles between the 3 -spaces $\mathbf{m}_{b_{3}, c_{3}, c_{2}}, \mathbf{m}_{b_{3}^{\prime}, c_{3}^{\prime}, c_{2}^{\prime}}$ and the 3 -space $\hat{\mathbf{m}}=\mathbf{m}_{0,0,0}$ are the same with respect to the Cartan-Killing form. As representatives of the isomorphism classes of the Bol loops $L_{b_{3}, c_{3}, c_{2}}$ may be chosen the Bol loops $\hat{L}_{d}=L_{d, 0,0}$ for $d \in[0,1)$. 
The pseudo-euclidean space loop $(\hat{L}, *)$ has a representation on the manifold $\Psi$ of euclidean planes in the 3 -dimensional pseudo-euclidean geometry $\mathcal{R}$ with the multiplication is given by the formula (**).

\section{Construction of 3-dimensional differentiab- le loops within the pseudo-euclidean space}

Let $\mathcal{R}$ be the 3 -dimensional pseudo-euclidean affine space. We embed the affine space $\mathbb{R}^{3}$ into the projective space $P_{3}(\mathbb{R})$ such that $(x, y, z) \mapsto$ $k(1, x, y, z), k \in \mathbb{R} \backslash\{0\}$. Then the projective plane $E$ at infinity consists of the points $\{k(0, x, y, z) \mid x, y, z \in \mathbb{R}, k \in \mathbb{R} \backslash\{0\}\}$. The cone $x^{2}+y^{2}-$ $z^{2}=0$ of the affine space $\mathbb{R}^{3}$ intersects the plane $E$ in the conic given by $C:\left\{k(0, x, y, z) \mid x^{2}+y^{2}=z^{2}, k(0, x, y, z) \neq(0,0,0,0)\right\}$. The conic $C$ divides the points of $E \backslash C$ in two regions $R_{1}, R_{2}$. The points $(0, x, y, z)$ of $R_{1}$ respectively of $R_{2}$ are characterized by the property $x^{2}+y^{2}<z^{2}$, respectively $x^{2}+y^{2}>z^{2}$.

Let $\Psi$ be the manifold of planes which intersect the plane $E$ in lines contained in the region $R_{2}$, i.e. in lines which do not meet $C$. There is a polarity $\pi$ of $E$ such that the absolute points of $\pi$ are the points of the conic $C$. The polarity $\pi$ interchanges the points of $R_{1}$ with the lines of $R_{2}$. We may assume that the connected component of the motion group of $G=P S L_{2}(\mathbb{R}) \ltimes \mathbb{R}^{3}$ on $\mathcal{R}$ is given by the Lorentz transformations. Since any translation of $\mathbb{R}^{3}$ leaves $E$ pointwise fixed the group induced by $G$ on $E$ is isomorphic to $P S L_{2}(\mathbb{R})$ and it is already induced by the stabilizer $\Gamma$ of $(0,0,0)$ in $G$.

Let $\Sigma$ be the submanifold of $\Gamma \cong P S L_{2}(\mathbb{R})$ which is the image of the section $\sigma_{1}$ defining the hyperbolic plane loop (cf. $\sigma_{1}$ before Theorem 6 ) and let $\Lambda$ be a 1-dimensional translation group leaving a line $S$ invariant which intersects any plane of $\Psi$ in precisely one point and contains the point $(0,0,0)$. We show that the product $\Theta=\Lambda \Sigma$ acts sharply transitively on the planes of $\Psi$ and may be taken as the image of a section for a differentiable 3-dimensional loop $L_{\Lambda}$ having $G$ as the group topologically generated by its left translations. Let $D_{1}, D_{2}$ be planes belonging to $\Psi$. We show that there is precisely an element $\rho=\omega \delta$ with $\delta \in \Sigma$ and $\omega \in \Lambda$ such that $\rho\left(D_{1}\right)=(\omega \delta)\left(D_{1}\right)=D_{2}$. Let $W_{1}=D_{1} \cap E$ and $W_{2}=D_{2} \cap E$. The lines $W_{1}$ and $W_{2}$ are contained in the region $R_{2}$. Hence $\pi\left(W_{1}\right)$ and $\pi\left(W_{2}\right)$ are points in $R_{1}$. In $\Sigma$ there is precisely one element $\delta$ such that $\delta\left(\pi\left(W_{1}\right)\right)=\pi\left(W_{2}\right)$. But then $\delta\left(W_{1}\right)=W_{2}$ and $\delta\left(D_{1}\right)$ is parallel to $D_{2}$. Let $s_{1}=D_{1} \cap S$ and $s_{2}=D_{2} \cap S$. Then there exists precisely one translation $\omega$ in $\Lambda$ with $\omega\left(s_{1}\right)=s_{2}$ and $\omega \delta\left(D_{1}\right)=D_{2}$. Let $P$ be a plane of $\Psi$ containing the point $(0,0,0)$ and $H$ be the stabilizer of 
$P$ in $G$. Then for the manifold $\Theta$, which topologically generates $G$, one has $\Theta \cap H=\{1\}$. Let $J=P \cap E$ and $L(P)$ be the line joining the points $(0,0,0)$ and $\pi(J)$. This line intersects any plane of $\Psi$ in precisely one points. Let $\Lambda_{L(P)}$ be the 1-dimensional translation group leaving the line $L(P)$ invariant. The multiplication for the loop $L_{\Lambda_{L(P)}}$ with the identity $P$ may be defined as follows:

$$
(X, Y) \mapsto \omega_{\left(\tau_{J, Q}(P), X\right)}\left(\tau_{J, Q}(Y)\right): \Psi \times \Psi \rightarrow \Psi
$$

where $\tau_{J, Q}$ is the unique element in $\Sigma$ mapping $J$ onto the line $Q=X \cap E$ and $\omega_{\left(\tau_{J, Q}(P), X\right)}$ is the unique translation in $\Lambda_{L(P)}$ mapping the plane $\tau_{J, Q}(P)$ onto $X$.

Let $K$ be the 1-dimensional compact subgroup of $\Gamma$ which leaves the line $J$ invariant. Then $K$ fixes $\pi(J)$ and leaves the line $L(P)$ pointwise invariant. Since $\Sigma \subset \Gamma$ is the image of a section corresponding to the hyperbolic plane loop with the identity $\pi(J)$ we have $g \Sigma g^{-1}=\Sigma$ and $g \Lambda_{L(P)} g^{-1}=\Lambda_{L(P)}$ for all $g \in K$. Hence the loop $L_{\Lambda_{L(P)}}$ has a 1-dimensional compact group of automorphisms.

Let $\Lambda^{*}$ be a 1-dimensional translation group such that the lines $S$ which are invariant under $\Lambda^{*}$ intersect any plane of $\Psi$, but $S \cap E \neq \pi(J)$. Then the loop $L_{\Lambda^{*}}$ with identity $P$ which corresponds to the section $\Lambda^{*} \Sigma$ and to the stabilizer $H$ is not isomorphic to the loop $L_{\Lambda_{L(P)}}$. This can be seen in the following way: The group $K \subseteq H$ does not leave $S$ invariant and hence does not normalize $\Lambda^{*}$. It follows that $g \Theta g^{-1}=g \Lambda^{*} \Sigma g^{-1}=\left(g \Lambda^{*} g^{-1}\right) g \Sigma g^{-1}=$ $\left(g \Lambda^{*} g^{-1}\right) \Sigma \neq \Theta$ and $K$ does not consists of automorphisms of the loop $L_{\Lambda^{*}}$. Every loop $L_{g^{-1} \Lambda^{*} g}$ with $g \in K$ is isomorphic to $L_{\Lambda^{*}}$.

No loop $L_{\Lambda}$ is a Bol loop since otherwise for elements $1 \neq \lambda \in \Lambda$ and $1 \neq$ $\rho \in \Sigma$ the element $(\lambda \rho) \cdot 1 \cdot \lambda \rho$ should be in $\Lambda \Sigma$. But one has $\lambda \rho \lambda \rho=$ $\lambda \rho \lambda \rho^{-1} \rho^{2} \notin \Lambda \Sigma$ since $\lambda \rho \lambda \rho^{-1} \notin \Lambda$. The submanifold $\Theta$ is the image of a differentiable section. Hence the multiplication of $L_{\Lambda}$ as well as the mappings $(a, b) \mapsto a \backslash b: L_{\Lambda} \times L_{\Lambda} \rightarrow L_{\Lambda}$ are differentiable. Let $D_{1}$ and $D_{2}$ be elements of $\Lambda_{L}$ and $D_{i} \cap E=W_{i}$ for $i=1,2$. Since the mapping $\left(W_{1}, W_{2}\right) \mapsto \tau_{J, Q} \in$ $\Sigma$ with $\tau_{J, Q}\left(W_{1}\right)=W_{2}$ as well as for parallel planes $D_{1}$ and $D_{2}$ the map $\left(D_{1}, D_{2}\right) \rightarrow \omega_{D_{1}, D_{2}} \in \Lambda$ with $\omega_{D_{1}, D_{2}}\left(D_{1}\right)=D_{2}$ are differentiable any loop $L_{\Lambda}$ is differentiable.

\section{References}

[1] T. Asoh, On smooth SL(2, C) actions on 3-manifolds, Osaka J. Math. 24 (1987), 271-298. 
[2] W. Benz, Geometrische transformationen unter besonderer Berücksichtigung der Lorenztransformationen, BI Wissenschaftverlag Mannheim, Leipzig, Wien, Zürich 1992.

[3] W. Fenchel, Elementary geometry in hyperbolic space, Walter de Gruyter, Berlin, New York 1989.

[4] P.M.D. Furness, Locally symmetric structures on surfaces, Bull. London Math. Soc. 8 (1976), 44-48.

[5] A.N. Grishkov, Three aspects of the exponential map, A.G. Pinus, Algebra and model theory. Novosibirsk State Technical University, 50-60 (1999).

[6] J. Hilgert and K. H. Hofmann, Old and new on SL(2), Manuscr. Math. 54 (1985), 17-52.

[7] K. Iwasawa, On some types of topological groups, Ann. Math. 50 (1949), 507-558.

[8] F.S. Kerdman, Moufang loops in the large (Russian). Algebra i Logika 18 (1979), 523-555.

[9] H. Kiechle, Theory of K-Loops, Lecture Notes in Mathematics. 1778. Springer-Verlag, Berlin, 2002.

[10] F. Klein, Vorlesungen über nicht-euklidische Geometrie, Die Grundlehren der mathematischen Wissenschaften, Verlag von Julius Springer, 1928.

[11] S. Kobayashi and K. Nomizu, Foundations of differential geometry, Vol I and Vol II, Wiley Interscience Publishers, New York, London, Sydney 1963, 1969.

[12] E.N. Kuz'min, The connection between Malcev algebras and analytic Moufang loops (Russian). Algebra i Logika 10 (1971), no.1, 3-22.

[13] W.G. Lister, A structure theory of Lie triple systems, Trans. Amer. Math. Soc. 72 (1952), 217-242.

[14] O. Loos, Symmetric Spaces, Vol I and Vol II. Benjamin, New York 1969, 1970.

[15] R. Löwen, Classification of 4-dimensional symmetric planes, Math. Z. 167 (1979), 137-159. 
[16] P.O. Miheev and L.V. Sabinin, Quasigroups and Differential Geometry, Chapter XII in Quasigroups and Loops:Theory and Applications (O. Chein, H.O. Pflugfelder and J.D.H. Smith), Sigma Series in Pure Math. 8, Heldermann-Verlag, Berlin, 1990, 357-430.

[17] P.T. Nagy, Extension of local loop isomorphism, Monatsh. Math. 112 (1991), 221-225.

[18] P.T. Nagy and K. Strambach, Loops in groups theory and Lie theory, de Gruyter Expositions in Mathematics. 35. Berlin, New York, 2002.

[19] L.V. Sabinin, Smooth quasigroups and loops, Math. Appl. 492, Kluwer Academic Publishers, Dordrecht 1999.

[20] J. Tits, Liesche Gruppen und Algebren, Hochschultext, Springer-Verlag, Berlin, Heidelberg, New York, Tokyo 1983.

[21] A. Vanzurová, Sabinin's method of classification of local Bol loops, Suppl. Rendiconti Circolo Matematico di Palermo, II. Ser. 59 (1999), 209-220.

Mathematisches Institut der Universität Erlangen-Nürnberg

Bismarckstr. $1 \frac{1}{2}$, D-91054 Erlangen, Germany

e-mail: figula@mi.uni-erlangen.de

and

Institute of Mathematics, University of Debrecen

P.O.B. 12, H-4010 Debrecen, Hungary

e-mail: figula@math.klte.hu 